\title{
FORBIDDEN INTEGER RATIOS OF CONSECUTIVE POWER SUMS
}

\author{
IOULIA N. BAOULINA AND PIETER MOREE \\ To the memory of Prof. Wolfgang Schwarz
}

\begin{abstract}
Let $S_{k}(m):=1^{k}+2^{k}+\cdots+(m-1)^{k}$ denote a power sum. In 2011 Bernd Kellner formulated the conjecture that for $m \geq 4$ the ratio $S_{k}(m+1) / S_{k}(m)$ of two consecutive power sums is never an integer. We will develop some techniques that allow one to exclude many integers $\rho$ as a ratio and combine them to exclude the integers $3 \leq \rho \leq 1501$ and, assuming a conjecture on irregular primes to be true, a set of density 1 of ratios $\rho$. To exclude a ratio $\rho$ one has to show that the Erdős-Moser type equation $(\rho-1) S_{k}(m)=m^{k}$ has no non-trivial solutions.
\end{abstract}

\section{INTRODUCTION}

Power sums have fascinated mathematicians for centuries. In this paper we consider some Diophantine equations involving power sums, of which the Erdős-Moser equation

$$
1^{k}+2^{k}+\cdots+(m-2)^{k}+(m-1)^{k}=m^{k}
$$

is typical and the most famous one. This equation has the obvious solution $(m, k)=$ $(3,1)$ and conjecturally no other solutions exist (this conjecture was formulated around 1950 by Paul Erdős in a letter to Leo Moser). Leo Moser [22], using only elementary number theory, established the following result.

Theorem 1 (Leo Moser, 1953). If $(m, k)$ is a solution of (1) with $k \geq 2$, then $m>10^{10^{6}}$.

For the shortest proof of this result presently known, we refer to Moree [18].

Using very different techniques, namely continued fractions and a many decimal computation of $\log 2$, Gallot et al. [7] established the current world record:

Theorem 2 (Gallot et al., 2011). If an integer pair $(m, k)$ with $k \geq 2$ satisfies (1), then

$$
m>2.7139 \cdot 10^{1667658416} \text {. }
$$

The bound $m>10^{10^{10}}$ seems feasible, but requires somewhat better computer resources than the authors of [7] had at their disposal.

Let $S_{k}(m):=\sum_{j=1}^{m-1} j^{k}$ be the sum of the first $m-1$ consecutive $k$ th powers. In this notation we can rewrite (11) as

$$
S_{k}(m)=m^{k} .
$$

Date: October 22, 2015.

2000 Mathematics Subject Classification. 11D61, 11A07. 
In the literature also the generalized Erdös-Moser conjecture is considered. The strongest result to date is due to the second author [19] who proved the following.

Theorem 3. For a fixed positive integer a, the equation $S_{k}(m)=a m^{k}$ has no integer solutions $(m, k)$ with

$$
k \geq 2, \quad m<\max \left(10^{9 \cdot 10^{6}}, a \cdot 10^{28}\right) .
$$

Interestingly, the method of Gallot et al. allows one only to deal with a specific value of $a$, for a general $a$ only the elementary method of Moser is available.

Kellner [11] conjectured that if $k$ and $m$ are positive integers with $m \geq 3$, the ratio $S_{k}(m+1) / S_{k}(m)$ is an integer iff $(m, k) \in\{(3,1),(3,3)\}$. Noting that $S_{k}(m+1)=$ $S_{k}(m)+m^{k}$, one observes that this conjecture is equivalent with the following one.

Conjecture 1 (Kellner-Erdös-Moser). Let $m \geq 3$. We have

$$
a S_{k}(m)=m^{k}
$$

iff $(a, m, k) \in\{(1,3,1),(3,3,3)\}$.

If this conjecture holds true, then obviously so does the Erdös-Moser conjecture. However, whereas the Erdős-Moser conjecture is open, we are able to establish the unsolvability of (3) for many integers $a$.

Theorem 4. If a has a regular prime as divisor or $2 \leq a \leq 1500$, then the equation $a S_{k}(m)=m^{k}$ has no solution with $m \geq 4$.

(We consider 2 to be a regular prime, see Section 2 for details.) The first restriction on $a$ is not very difficult to prove, but powerful in its consequences. Assume that there exists a real number $\delta<1$ such that the number of irregular primes $p \leq x$ is bounded above by $\delta x / \log x$ as $x \rightarrow \infty$. It then follows (see Section 21) that, for a set of integers $a$ of density $1, a S_{k}(m)=m^{k}$ has no solution with $m \geq 4$. The first restriction implies that in order to exclude the $a$ in the range $2 \leq a \leq 1500$, one has to exclude $a=37^{2}$ and all irregular primes in this interval. These $a$ can be dealt with using various technical and not very general necessary conditions for (3) to be solvable.

In case we are not able to exclude a square-free $a$, we are able to show that if (3) holds, then both $k$ and $m$ are large.

Theorem 5. Suppose that $a S_{k}(m)=m^{k}, m \geq 4$ and $a$ is square-free, then both $k$ and $m$ exceed $3.44 \cdot 10^{82}$.

We like to point out that for solutions with $m \equiv 1(\bmod 3)$ or $m \equiv 1(\bmod 30)$ much larger lower bounds hold true (see Theorem 7 ).

For a fixed integer $a \geq 1$ it is not known whether there are finitely many solutions $(m, k)$ of (3) or not. In this direction we can only contribute the following modest result.

Proposition 1. Let $\left(m_{1}, k_{1}\right)$ and $\left(m_{2}, k_{2}\right)$ be different solutions of $a S_{k}(m)=m^{k}$. Then $m_{1} \neq m_{2}$ and $k_{1} \neq k_{2}$. 
A final approach of dealing with equation (3) is to try to prove that $k$ is divisible by 120 say. Once established, there are many options of how to get an even bigger number to divide $k$. A cascade of ways how to proceed further arises and it seems very likely then that also in this case $a+1$ cannot occur as a ratio. We demonstrate this cascade process in Section 7. Paul Tegelaar [26] jokingly called this 'the method of infinite ascent'.

After discussing some basic material on Bernoulli numbers and power sums in Section 2, we obtain a crucial result (Theorem 6) relating Bernoulli numbers and solutions of the Kellner-Erdös-Moser equation in Section 3. Integers $a$ that are a product of irregular primes cannot be immediately excluded, and for these one can use helpful pairs, see Section 4. They allow one to rule out that $k \equiv c(\bmod d)$ for many even integers $c \geq 2$ and $d$. In Section 5 we demonstrate with both an easy and a difficult example how to exclude a given integer ratio $\rho$. Table 3 illustrates in a compact way how to show that $3 \leq \rho \leq 1501$ are forbidden ratios. Some ratios are clearly much easier to exclude than others and this is discussed in Section 6. In Section 7 we discuss how to show that a given integer divides $k$. In Section 8 we reason in the way of Moser to derive lower bounds for $k$ and $m$ in case $a$ is squarefree. In Section 9 proofs of the new results announced above are given. These are mainly based on work done in earlier sections. In the final section, Section 10, some further properties of potential solutions of the Kellner-Erdös-Moser equation are derived. The proof that the integers $2 \leq a \leq 1500$ are forbidden makes use of Tables 2 and 3 .

A survey of earlier work on Erdös-Moser type conjectures can be found in Moree [19], also see [3, Chapter 8], for an expository account of the work of Gallot et al. [7].

\section{Preliminaries on Bernoulli numbers AND POWER Sums}

Lemma 1 (Carlitz-von Staudt). Let $k$ and $m$ be positive integers, then

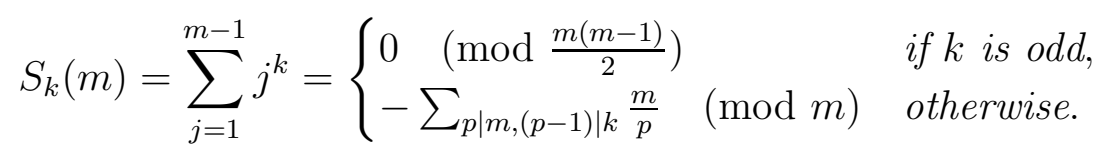

This result, with some small error (cf. Moree [16]), was published in 1961 by Carlitz. For an easy reproof of the above result, see Moree [18].

Recall that the Bernoulli numbers $B_{k}$ are defined by the power series

$$
\frac{t}{e^{t}-1}=\sum_{k=0}^{\infty} \frac{B_{k} t^{k}}{k !} \text {. }
$$

They are rational numbers and can be written as $B_{k}=U_{k} / V_{k}$, with $V_{k}>0$ and $\operatorname{gcd}\left(U_{k}, V_{k}\right)=1$. One has $B_{0}=1, B_{1}=-1 / 2, B_{2}=1 / 6$ and $B_{2 j+1}=0$ for $j \geq 1$.

In the next four lemmas, we record some well-known facts about the Bernoulli numbers (see [8, Chapter 15]).

Lemma 2 (von Staudt-Clausen). If $k \geq 2$ is even, then $V_{k}=\prod_{(p-1) \mid k} p$.

Lemma 3 (Kummer congruence). Let $k \geq 2$ be even and $p$ be a prime with $(p-1) \nmid k$. If $k \equiv r(\bmod p-1)$, then $B_{k} / k \equiv B_{r} / r(\bmod p)$. 
Lemma 4. For any integers $k \geq 1$ and $m \geq 2$,

$$
S_{k}(m)=\sum_{j=0}^{k}\left(\begin{array}{l}
k \\
j
\end{array}\right) B_{k-j} \frac{m^{j+1}}{j+1} .
$$

Lemma 5 (Voronoi congruence). Let $k$ and $m$ be positive integers, where $m \geq 2$ and $k \geq 2$ is even, then $V_{k} S_{k}(m) \equiv U_{k} m\left(\bmod m^{2}\right)$.

The following lemma gives a refinement of the Voronoi congruence (see [10, Proposition 8.5]).

Lemma 6. Let $k$ and $m$ be positive integers, where $m \geq 2$ and $k \geq 6$ is even. If $m \mid U_{k}$ then $V_{k} S_{k}(m) \equiv U_{k} m\left(\bmod m^{3}\right)$.

A prime $p$ will be called regular if it does not divide any of the numerators $U_{r}$ with even $r \leq p-3$, otherwise it is said to be irregular. The pairs $(r, p)$ with $p \mid U_{r}$ and even $r \leq p-3$ are called irregular pairs. At a first glance this looks like a strange definition, but by celebrated work of Kummer (1850) [12] can be reformulated as: a prime $p$ is irregular if and only if it divides the class number of $\mathbb{Q}\left(\zeta_{p}\right)$. The first few irregular primes are $37,59,67,101,103,131,149, \ldots$. It is known that there are infinitely many irregular primes, cf. Carlitz [6]. It is not known whether there are infinitely many regular primes. Let $\pi_{\iota}(x)$ denote the number of irregular primes $p \leq x$. Recently Luca et al. [14, Theorem 1] showed that

$$
\pi_{\iota}(x) \geq(1+o(1)) \frac{\log \log x}{\log \log \log x}, \quad x \rightarrow \infty .
$$

Conjecturally, cf. Siegel [25], and in good agreement with numerical work, we should have

$$
\pi_{\iota}(x) \sim\left(1-\frac{1}{\sqrt{e}}\right) \pi(x) \approx 0.3935 \frac{x}{\log x} .
$$

Let $N_{\iota}(x)$ denote the number of integers $n \leq x$ that are composed only of irregular primes. If we assume that

$$
\pi_{\iota}(x) \sim \delta \frac{x}{\log x}, \quad 0<\delta<1,
$$

then by Moree [20, Theorem 1] we have $N_{\iota}(x) \sim c x \log ^{\delta-1} x$ as $x \rightarrow \infty$, with $c$ a positive real constant. (Kummer conjectured that $\delta=1 / 2$.) The latter result is of Wirsing type (cf. Schwarz and Spilker [24, 65-76]).

For more results on Bernoulli numbers see e.g. the book by Arakawa et al. [1.

\section{The KeLlner-Erdős-Moser COnJeCture}

In this section, we will use properties of Bernoulli numbers to study the nontrivial solutions of the equation $a S_{k}(m)=m^{k}$. This will then lead us to establish Theorem 6. As a bonus we will conclude that if $a S_{k}(m)=m^{k}$ has non-trivial solutions, then $a$ must be either 1 or a product of irregular primes.

First assume that $m=2$. Then $a=2^{k}$. Next assume that $m=3$. Then we must have $a\left(1+2^{k}\right)=3^{k}$ and hence $a=3^{e}$ for some $e \leq k$. It follows that 
$1+2^{k}=3^{k-e}$. This Diophantine equation was already solved by the famous medieval astronomer Levi ben Gerson (1288-1344), alias Leo Hebraeus, who showed that 8 and 9 are the only consecutive integers in the sequence of powers of 2 and 3 , see Ribenboim [23, pp. 124-125]. This leads to the solutions $(e, k) \in\{(0,1),(1,3)\}$ and hence $(a, m, k) \in\{(1,3,1),(3,3,3)\}$. Now assume that $m \geq 4$ and $k$ is odd. Then by Lemma 1 we find that $m(m-1) / 2$ divides $m^{k}$, which is impossible. We infer that, to establish Conjecture 1, it is enough to establish the following conjecture.

Conjecture 2. The set

$$
\mathcal{A}=\left\{a \geq 1: a S_{k}(m)=m^{k} \text { has a solution with } m \geq 4 \text { and } k \text { even }\right\}
$$

is empty.

Lemma 7. Suppose that $a S_{k}(m)=m^{k}$ with $m \geq 4$ and $k$ even. If $p$ is a prime dividing $m$, then $(p-1) \nmid k$.

Proof. Assume that $p \mid m$ and $(p-1) \mid k$. Let $p^{e} \| m$ and $p^{f} \| a$, where $e \geq 1$, $f \geq 0$. Using Lemma 1 we find that $S_{k}(m) \equiv \frac{m}{p^{e}} S_{k}\left(p^{e}\right) \equiv-\frac{m}{p}\left(\bmod p^{e}\right)$. Hence $a S_{k}(m) \equiv-\frac{a m}{p}\left(\bmod p^{e+f}\right)$, and so $m^{k} \equiv-\frac{a m}{p}\left(\bmod p^{e+f}\right)$. Since $p^{e+f-1} \| \frac{a m}{p}$, we deduce that $p^{e+f-1} \| m^{k}$, and thus $f=(k-1) e+1 \geq k$. Then $a \geq p^{k} \geq 2^{k}$ and $a S_{k}(m)>2^{k}(m-1)^{k}>m^{k}$, which contradicts the fact that $a S_{k}(m)=m^{k}$.

Corollary 1. Suppose that $a S_{k}(m)=m^{k}$ with $m \geq 4$ and $k$ even, then we have $\operatorname{gcd}(m, 6)=1$.

Corollary 2. If $\operatorname{gcd}(a, 6) \neq 1$, then $a \notin \mathcal{A}$.

Lemma 8. Suppose that $a S_{k}(m)=m^{k}$ with $m \geq 4$ and $k$ even. Suppose that a has no prime divisor $p$ satisfying $p<2^{s}$. Then $a \mid m^{\lceil k / s\rceil-1}$.

Proof. Assume that $a \nmid m^{\lceil k / s\rceil-1}$. Since each prime divisor of $a$ divides $m$, there exists a prime $p$ such that $p^{\lceil k / s\rceil} \mid a$. By assumption $p \geq 2^{s}$. Then $a \geq\left(2^{s}\right)^{\lceil k / s\rceil} \geq 2^{k}$ and $a S_{k}(m) \geq 2^{k}(m-1)^{k}>m^{k}$, which is a contradiction.

On combining the latter lemma and Corollary 2 we obtain the following result.

Corollary 3. Suppose that $a S_{k}(m)=m^{k}$ with $m \geq 4$ and $k$ even, then $a \mid m^{(k-2) / 2}$.

Lemma 9. Suppose that $a S_{k}(m)=m^{k}$ with $m \geq 4$ and $k$ even, then $m \mid U_{k}$.

Proof. Multiplying the Voronoi congruence by $a$ and using the fact that $a S_{k}(m)=$ $m^{k}$, we deduce that $V_{k} m^{k} \equiv U_{k} a m\left(\bmod a m^{2}\right)$. Since $m^{2} \mid m^{(k+2) / 2}$ and, by Corollary [3, $a \mid m^{(k-2) / 2}$, we have $a m^{2} \mid U_{k} a m$, that is $m \mid U_{k}$.

Corollary 4. Suppose that $a S_{k}(m)=m^{k}$ with $m \geq 4$ and $k$ even, then $k \geq 10$.

Lemma 10. Suppose that $a S_{k}(m)=m^{k}$ with $m \geq 4$ and $k$ even, then $m^{2} \mid U_{k}$.

Proof. By Corollary 4, $k \geq 10$. Using Lemma 6] instead of the Voronoi congruence and proceeding then by the same argument as in the proof of Lemma 9, we deduce that $a m^{3} \mid U_{k} a m$, and so $m^{2} \mid U_{k}$.

Since $U_{k}$ is square-free for any even $k<50$, we have 
Corollary 5. Suppose that $a S_{k}(m)=m^{k}$ with $m \geq 4$ and $k$ even, then $k \geq 50$.

In case $a=1$ the next result is Lemma 10 of [21].

Theorem 6. Suppose that $a S_{k}(m)=m^{k}$ with $m \geq 4$ and even $k$. Let $p$ be a prime dividing $m$. Then

(a) $p$ is an irregular prime;

(b) $k \not \equiv 0,2,4,6,8,10,14(\bmod p-1)$;

(c) $\operatorname{ord}_{p}\left(B_{k} / k\right) \geq 2 \operatorname{ord}_{p} m \geq 2$;

(d) $k \equiv r(\bmod p-1)$ for some irregular pair $(r, p)$.

Proof. By Corollary 11 and Lemmas 7 and 9 we see that $p \geq 5,(p-1) \nmid k$ and $p \mid U_{k}$. If $p \nmid k$, then $\operatorname{ord}_{p}\left(B_{k} / k\right)>0$ and $p$ is irregular. Now assume that $p \mid k$, i.e., $\operatorname{ord}_{p} k \geq 1$. In view of Corollary 4 we have $k \geq 10$. Further

$$
S_{k}(m)=B_{k} m+\frac{k(k-1)}{6} B_{k-2} m^{3}+\sum_{j=4}^{k}\left(\begin{array}{c}
k \\
j
\end{array}\right) B_{k-j} \frac{m^{j+1}}{j+1} .
$$

Hence

$$
\frac{m^{k-1}}{a k}=\frac{B_{k}}{k}+\frac{k-1}{6} B_{k-2} m^{2}+m^{2} \sum_{j=4}^{k} \frac{(k-1) !}{(k-j) !} B_{k-j} \frac{m^{j-2}}{(j+1) !} .
$$

By Lemma 2 we have $\operatorname{ord}_{p} V_{k-2} \leq 1$, and hence

$$
\operatorname{ord}_{p}\left(\frac{k-1}{6} B_{k-2} m^{2}\right) \geq 2 \operatorname{ord}_{p} m-\operatorname{ord}_{p} V_{k-2} \geq 1 .
$$

Further, for $j=4,5, \ldots, k$,

$$
\operatorname{ord}_{p}\left(\frac{m^{j-2}}{(j+1) !}\right)=(j-2) \operatorname{ord}_{p} m-\frac{j+1-\sigma_{p}(j+1)}{p-1} \geq j-2-\frac{j}{4} \geq 1,
$$

where $\sigma_{p}(j+1)$ denotes the sum of the digits of $j+1$ written in the base $p$. Therefore

$$
\operatorname{ord}_{p}\left(m^{2} \sum_{j=4}^{k} \frac{(k-1) !}{(k-j) !} B_{k-j} \frac{m^{j-2}}{(j+1) !}\right) \geq 2 \operatorname{ord}_{p} m \geq 2 .
$$

Note that

$$
\begin{aligned}
\operatorname{ord}_{p}\left(\frac{m^{k / 2}}{k}\right) & =\frac{k}{2} \operatorname{ord}_{p} m-\operatorname{ord}_{p} k \geq \frac{1}{2} p^{\operatorname{ord}_{p} k}-\operatorname{ord}_{p} k \\
& >2^{2 \operatorname{ord}_{p} k-1}-\operatorname{ord}_{p} k \geq \operatorname{ord}_{p} k \geq 1 .
\end{aligned}
$$

Using Corollary 3 we obtain

$$
\operatorname{ord}_{p}\left(\frac{m^{(k-2) / 2}}{a}\right) \geq 0
$$

It follows from (8) and (9) that

$$
\operatorname{ord}_{p}\left(\frac{m^{k-1}}{a k}\right)=\operatorname{ord}_{p}\left(\frac{m^{(k-2) / 2}}{a} \cdot \frac{m^{k / 2}}{k}\right) \geq 1 .
$$


Combining (5) - (17) and (10), we deduce that $\operatorname{ord}_{p}\left(B_{k} / k\right)>0$, and so $p$ is irregular. This completes the proof of part (a). Part (b) is a consequence of part (a), Lemma 7 and the Kummer congruence. In the case $p \nmid k$, part (c) follows from Lemma 10, Now assume that $p \mid k$. By part (a), $p$ is an irregular prime, and so $p \geq 37>2^{5}$. On combining Lemma 8 with $s=5$ and Corollary [5, (9) is sharpened to

$$
\operatorname{ord}_{p}\left(\frac{m^{(k-2) / 2}}{a}\right)>2 \operatorname{ord}_{p} m \text {. }
$$

Combining the latter estimate with (8) gives

$$
\operatorname{ord}_{p}\left(\frac{m^{k-1}}{a k}\right)>2 \operatorname{ord}_{p} m .
$$

Further, by part (b) and the von Staudt-Clausen theorem, $\operatorname{ord}_{p} V_{k-2}=0$. Combining (5), (6), (7) and (11), we complete the proof of part (c). Part (d) is a direct consequence of part $(c)$, the fact that $(p-1) \nmid k$, and the Kummer congruence.

Corollary 6. If a has a regular prime divisor, then $a \notin \mathcal{A}$.

Corollary 7. Let $p_{1}$ and $p_{2}$ be distinct irregular prime divisors of a. Assume that for every pair $\left(r_{1}, p_{1}\right),\left(r_{2}, p_{2}\right)$ of irregular pairs we have $\operatorname{gcd}\left(p_{1}-1, p_{2}-1\right) \nmid\left(r_{1}-r_{2}\right)$. Then $a \notin \mathcal{A}$.

Example. Suppose that $37 \cdot 379 \mid a$. If $a \in \mathcal{A}$ then $a S_{k}(m)=m^{k}$ with $m \geq 4$ and $k$ even and both 37 and 379 must divide $m$. There is one irregular pair $(32,37)$ corresponding to 37 and two irregular pairs $(100,379)$ and $(174,379)$ corresponding to 379 . By Theorem 6 (d), $k$ must be a simultaneous solution of the congruences $k \equiv$ $32(\bmod 36)$ and $k \equiv 100$ or $174(\bmod 378)$, which is impossible as $\operatorname{gcd}(36,378)=$ $18,18 \nmid(32-100)$ and $18 \nmid(32-174)$. Hence $a \notin \mathcal{A}$.

\section{Helpful pairs}

Helpful pairs will be used to show that certain ratios are forbidden (Section 5) and to show that certain numbers have to divide $k$ (Section 7). In both cases one has to exclude that $k$ is in certain congruence classes. In order to show that a certain ratio is forbidden, we have to exclude all the congruence classes with an appropriate modulus. In order to show that a certain even number $d$ divides $k$, we do this by excluding all the congruence classes $2 i(\bmod d)$ for $1 \leq i<d / 2$. If $p \mid a$ is an irregular prime, $d=p-1$, then by Theorem [6] we immediately exclude many congruence classes.

The exclusion of a congruence is achieved by a helpful pair and the procedure is described just after the proof of the crucial Lemma 11.

Definition 1. For a positive integer a let us call a pair $(t, q)_{a}$ with $q$ a prime and $2 \leq t \leq q-3$ even to be helpful if $q \nmid a$ and $a S_{t}(c) \not \equiv c^{t}(\bmod q)$ for every integer $c$ satisfying $1 \leq c \leq q-1$. If $q$ is an irregular prime, we require in addition that $(t, q)$ should not be an irregular pair.

Proposition 2. Let $q \geq 5$ be a prime and a be a positive integer. Then $(2, q)_{a}$ is a helpful pair if and only if $\left(\frac{a^{2}+36 a+36}{q}\right)=-1$. 
Proof. Note that $B_{2}=1 / 6$ and hence $(2, q)$ cannot be an irregular pair. Since $S_{2}(c)=\left(2 c^{3}-3 c^{2}+c\right) / 6$, we see that $(2, q)_{a}$ is a helpful pair if and only if $q \nmid a$ and $a\left(2 c^{3}-3 c^{2}+c\right) \not \equiv 6 c^{2}(\bmod q)$ for $c=1, \ldots, q-1$, that is, if and only if $a\left(2 c^{2}-3 c+1\right) \not \equiv 6 c(\bmod q)$ for $c=0, \ldots, q-1$, i.e., if and only if we have $\left(\frac{9(a+2)^{2}-8 a^{2}}{q}\right)=\left(\frac{a^{2}+36 a+36}{q}\right)=-1$.

Proposition 3. Let $q \geq 7$ be a prime with $\left(\frac{31}{q}\right)=-1$. Then $(4, q)_{q-2}$ is a helpful pair.

Proof. From $\left(\frac{31}{q}\right)=-1$ we deduce that $6 n^{2}+10 n-1 \not \equiv 0(\bmod q)$ for $n=1, \ldots, q-1$. This implies that $30(q-2) S_{4}(c)-30 c^{4} \equiv-2 c\left(6 c^{4}+10 c^{2}-1\right) \not \equiv 0(\bmod q)$ for $c=1, \ldots, q-1$. Since $B_{4}=-1 / 30,(4, q)$ is not an irregular pair. Thus $(4, q)_{q-2}$ is a helpful pair.

Lemma 11. Let $2 \leq t \leq q-3$ and $q$ be a prime. If $(t, q)_{a}$ is a helpful pair and $a S_{k}(m)=m^{k}$ with $k$ even, then we have $k \not \equiv t(\bmod q-1)$.

Proof. Assume that $k \equiv t(\bmod q-1)$. By Theorem 6 (d) we must have $q \nmid m$, for otherwise $(t, q)$ is an irregular pair, contradicting the definition of a helpful pair. Thus we can write $m=m_{0} q+b$ with $1 \leq b \leq q-1$. By Lemma 1 we have $q \mid S_{t}(q)$. We now find, modulo $q, S_{k}(m) \equiv S_{t}(m) \equiv m_{0} S_{t}(q)+S_{t}(b) \equiv S_{t}(b)$. Thus if (3) is satisfied we must have $a S_{t}(b) \equiv b^{t}(\bmod q)$. By the definition of a helpful pair this is impossible.

Ruling out congruence classes for $k$. The helpful pairs give us a chance to rule out $k$ that satisfy certain congruences of the form $k \equiv c(\bmod d)$ with $c \geq 2$ and $d$ even. We first list all primes $q \geq 5$ such that $q-1$ divides $d$. Denote these primes by $q_{1}, \ldots, q_{s}$. Let $t_{i}$ denote the least nonnegative integer congruent to $c$ modulo $q_{i}-1$. If one of the pairs $\left(t_{i}, q_{i}\right)_{a}$ is helpful, by Lemma 11 we have ruled out $k \equiv c(\bmod d)$. If this does not work, we multiply $d$ by an integer $\ell \geq 2$ (the lifting factor). Our original congruence is now replaced by $\ell$ congruences, $k \equiv c+j d$ $(\bmod \ell d), 0 \leq j<\ell$. For each of these congruences we now continue as before. In certain cases we find that each of the lifted congruences is ruled out by a helpful pair and then we are done. This situation is described in Proposition 4 below. If not all of the lifted congruences are excluded by helpful pairs, we can lift the bad congruences still further. The above procedure is not systematic and each stage the danger lurks that we get too many congruence classes we cannot exclude anymore.

Proposition 4. Let $p$ be an irregular prime dividing a. Assume that for every irregular pair $(r, p)$ there exists a positive integer $\ell_{r}$ such that for every $j=0,1, \ldots, \ell_{r}-1$ there is a helpful pair $\left(t_{j}, q_{j}\right)_{a}$ with $\left(q_{j}-1\right) \mid \ell_{r}(p-1)$ and $t_{j} \equiv r+j(p-1)\left(\bmod q_{j}-1\right)$. Then $a \notin \mathcal{A}$.

Proof. Since $p$ must divide $m$, Theorem $6(\mathrm{~d})$ yields $k \equiv r(\bmod p-1)$ for some irregular pair $(r, p)$. Hence there exists $j \in\left\{0,1, \ldots, \ell_{r}-1\right\}$ such that $k \equiv r+j(p-1)$ $\left(\bmod \ell_{r}(p-1)\right)$. Then we have $k \equiv t_{j}\left(\bmod q_{j}-1\right)$ for the helpful pair $\left(t_{j}, q_{j}\right)_{a}$, which contradicts Lemma 11 . 
Corollary 8. Under the conditions of Proposition 4 , we have ab $\notin \mathcal{A}$ for any positive integer $b \equiv 1(\bmod Q)$, where $Q$ denotes the least common multiple of all components $q_{j}$ of helpful pairs constructed for all irregular pairs $(r, p)$ corresponding to $p$.

\section{EXCLUDing A GIVEN RAtio $\rho$}

Let $\rho \geq 3$. Write $a=\rho-1$. If $a$ has no regular prime divisor, the only way we know to exclude $\rho$ is by using helpful pairs or invoking Corollary 7 . We demonstrate this with two examples (an example of the usage of Corollary 7 we already gave immediately following the statement of Corollary 7).

Easy example: $a=673$.

There are two irregular pairs $(408,673)$ and $(502,673)$ corresponding to 673 . Theorem 6 (d) yields $k \equiv 408$ or $502(\bmod 672)$. If $k \equiv 408(\bmod 672)$, then $k \equiv 8$ (mod 16). The latter is impossible since $(8,17)_{10}$ is a helpful pair by Table 2 . If $k \equiv 502(\bmod 672)$, then $k \equiv 2(\bmod 4)$, which is impossible as $(2,5)_{3}$ is a helpful pair by Table 2 .

Difficult example: $a=653$.

There is one irregular pair $(48,653)$ corresponding to 653 , and so $k \equiv 48(\bmod 652)$. We have $652=2^{2} \cdot 163$. There are no helpful pairs $(48, q)$ with $(q-1) \mid 652$. So we have to use a lifting factor $\ell$. It turns out that $\ell=4$ is a useful factor. So that is why we use it in the first step.

Step 1 . We have $k \equiv 48$ or 700 or 1352 or $2004(\bmod 2608)$. The case $k \equiv 48$ (mod 2608) is impossible, since $(48,2609)_{653}$ is a helpful pair. If $k \equiv 700$ or 1352 $(\bmod 2608)$, then $k \equiv 8$ or $12(\bmod 16)$, which is impossible as $(8,17)_{7}$ and $(12,17)_{7}$ are helpful pairs. Thus $k \equiv 2004(\bmod 2608)$.

Step 2. We have $k \equiv 2004$ or 4612 or 7220 or 9828 or $12436(\bmod 13040)$. If $k \equiv 4612$ or 7220 or $9828(\bmod 13040)$, then $k \equiv 12$ or 20 or $28(\bmod 40)$. From the fact that $(12,41)_{38},(20,41)_{38}$ and $(28,41)_{38}$ are helpful pairs we deduce that the latter congruence is impossible. Hence $k \equiv 2004$ or $12436(\bmod 13040)$.

Step 3. We have $k \equiv 2004$ or 12436 or 15044 or 25476 or 28084 or 38516 $(\bmod 39120)$. If $k \equiv 15044$ or $28084(\bmod 39120)$ then $k \equiv 4$ or $14(\bmod 30)$, which is impossible as $(4,31)_{2}$ and $(14,31)_{2}$ are helpful pairs. If $k \equiv 2004$ or 25476 $(\bmod 39120)$, then $k \equiv 24$ or $36(\bmod 60)$. The latter is impossible, since $(24,61)_{43}$ and $(36,61)_{43}$ are helpful pairs. The case $k \equiv 12436(\bmod 39120)$ implies $k \equiv 196$ $(\bmod 240)$, which is impossible as $(196,241)_{171}$ is a helpful pair. The remaining case $k \equiv 38516(\bmod 39120)$ is also impossible, since in this case $k \equiv 9176(\bmod 9780)$ and $(9176,9781)_{653}$ is a helpful pair.

Remark. Using helpful pairs, we can find some infinite families of forbidden ratios. For example, let $\rho=37^{s}+1$ for some positive integer $s$. The prime 37 is irregular and $(32,37)$ an irregular pair. If $37^{s} S_{k}(m)=m^{k}$ with $k$ even then, by Theorem 6 (d), we have $k \equiv 32(\bmod 36)$. This implies that $k \equiv 8(\bmod 12)$. Since $(8,13)_{37^{s}}$ is a helpful pair if and only if $37^{s} \equiv 1$ or 2 or 6 or 8 or $11(\bmod 13)$ (see Table 2$)$, 
we deduce that $\rho=37^{s}+1$ is a forbidden ratio for any $s \equiv 0$ or 1 or 7 or 9 or 11 $(\bmod 12)$.

\section{BAD RATIOS}

Table 3 gives a list of ratios we excluded and the helpful pairs used to do so. The attentive reader will notice that various ratios $\rho$ are apparently bad and difficult to exclude. These are related to $a=\rho-1$ that are of the form $(2 p+1)^{s}$ with $p$ a Sophie Germain prime. Recall that a prime $p$ is said to be a Sophie Germain prime if also $2 p+1$ is a prime. Heuristics suggests that there ought to be infinitely many Sophie Germain primes such that $2 p+1$ is an irregular prime.

Conjecture 3. There are infinitely many primes $p$ such that $2 p+1$ is an irregular prime.

Let $p$ be a prime such that $2 p+1$ is an irregular prime and let $(r, 2 p+1)$ be an irregular pair. In case we want to rule out $k \equiv r(\bmod 2 p)$ we are in bad shape to start with. We are directly forced here to use a lifting factor $\ell \geq 2$ (as the list of primes $5 \leq q<2 p+1$ with $(q-1) \mid 2 p$ is empty here). The next result shows that we are in even worse shape, since helpful pairs with primes $q=2 p u+1>6 p$ have to be used.

Proposition 5. Let $p$ be a prime such that $2 p+1$ is an irregular prime dividing $a$ and let $(r, 2 p+1)$ be an irregular pair. Let $\ell$ be a positive integer with $p \nmid \ell$, let $q_{0}, q_{1}, \ldots, q_{\ell-1}$ be odd primes with $\left(q_{j}-1\right) \mid 2 \ell$ (not necessarily distinct) and let $t_{0}, t_{1}, \ldots, t_{\ell-1}$ be positive integers satisfying the conditions $t_{j} \equiv r+2 p j\left(\bmod q_{j}-1\right)$, $0 \leq j \leq \ell-1$. Then at least one of the pairs $\left(t_{0}, q_{0}\right)_{a},\left(t_{1}, q_{1}\right)_{a}, \ldots,\left(t_{\ell-1}, q_{\ell-1}\right)_{a}$ is not a helpful pair.

Proof. Since $r$ is even and $p \nmid \ell$, there exists a $j$ with $0 \leq j \leq \ell-1$ such that $p j \equiv-r / 2(\bmod \ell)$. Hence $2 \ell \mid(r+2 p j)$. This implies that $\left(q_{j}-1\right) \mid t_{j}$, and so $\left(t_{j}, q_{j}\right)_{a}$ is not a helpful pair.

In case we are not able to exclude such a bad ratio, we might try at least to show that the $k$ of a solution has to be highly divisible. In the next section we demonstrate this for the bad ratio 6780 .

\section{Divisibility of $k$}

In this section, we consider the case $a=6779=2 \cdot 3389+1$ and show that for a non-trivial solution $k$ is divisible by a large number. We will present an heuristic argument here why we think that for this $a$ there are no solutions. We expect that a similar reasoning might work for other values of $a$ as well, once one can establish that a smallish number like 120 divides $k$.

We start by discussing a baby example.

\section{Proposition 6.}

(a) If $a \equiv 1$ or 2 or $3(\bmod 5)$, then $4 \mid k$.

(b) If $a \equiv 1$ or 3 or $5(\bmod 7)$, then $6 \mid k$. 
(c) If $a \equiv 6$ or $7(\bmod 11)$, then $10 \mid k$.

(d) If $a \equiv 2$ or 8 or $11(\bmod 13)$, then $12 \mid k$.

(e) If $a \equiv 1$ or $6(\bmod 13)$, then $6 \mid k$.

(f) If $a \equiv 1$ or $5(\bmod 11)$ and $a \equiv 15(\bmod 31)$, then $10 \mid k$.

Proof. If, e.g., $a \equiv 2(\bmod 13)$, we see from Table 2 that the pairs $(2,13)_{a},(4,13)_{a}$, $(6,13)_{a},(8,13)_{a}$ and $(10,13)_{a}$ are all helpful. The final assertion follows from glancing at an extended version of Table 2 .

Now let us consider a more serious example, with $\rho=6780$ a bad ratio.

Proposition 7. If $6779 S_{k}(m)=m^{k}$, then $2^{6} \cdot 3^{3} \cdot 5^{2} \cdot 7 \cdot 11 \cdot 13 \mid k$.

Proof. We start with the congruence $k \equiv 3994(\bmod 6778)$, which is a consequence of Theorem 6 (d) and the fact that there is only one irregular pair $(3994,6779)$ corresponding to 6779 .

Step 1 . We have $k \equiv 3994$ or 10772 or $17550(\bmod 20334)$. If $k \equiv 3994$ or 10772 $(\bmod 20334)$ then $k \equiv 2$ or $4(\bmod 6)$, which is impossible as $(2,7)_{3}$ and $(4,7)_{3}$ are helpful pairs. Hence $k \equiv 17550(\bmod 20334)$ and $2 \cdot 3 \mid k$.

Step 2 . We have $k \equiv 17550$ or 37884 or $58218(\bmod 61002)$. The case $k \equiv 58218$ $(\bmod 61002)$ is impossible, since in this case $k \equiv 6(\bmod 18)$ and $(6,19)_{15}$ is a helpful pair. Hence $k \equiv 17550$ or $37884(\bmod 61002)$.

Step 3. We have $k \equiv 17550$ or 37884 or 78552 or $98886(\bmod 122004)$. If $k \equiv$ 17550 or $98886(\bmod 122004)$, then $k \equiv 18$ or $30(\bmod 36)$, which is impossible as $(18,37)_{8}$ and $(30,37)_{8}$ are helpful pairs. Hence $k \equiv 37884$ or $78552(\bmod 122004)$.

Step 4. We have $k \equiv 37884$ or 78552 or 159888 or $200556(\bmod 244008)$. If $k \equiv 37884$ or 159888 or $200556(\bmod 244008)$, then $k \equiv 12$ or 36 or $48(\bmod 72)$, which is impossible as $(12,73)_{63},(36,73)_{63}$ and $(48,73)_{63}$ are helpful pairs. Hence $k \equiv 78552(\bmod 244008)$ and $2^{3} \cdot 3^{2} \mid k$.

Step 5 . We have $k \equiv 78552$ or $322560(\bmod 488016)$. In the case $k \equiv 78552$ $(\bmod 488016)$ we have $k \equiv 8(\bmod 16)$, which is impossible as $(8,17)_{13}$ is a helpful pair. Hence $k \equiv 322560(\bmod 488016)$ and $2^{4} \cdot 3^{2} \mid k$.

Step 6 . We have $k \equiv 322560$ or $810576(\bmod 976032)$. The case $k \equiv 810576$ $(\bmod 976032)$ is impossible, since in this case $k \equiv 48(\bmod 96)$ and $(48,97)_{86}$ is a helpful pair. Hence $k \equiv 322560(\bmod 976032)$ and $2^{5} \cdot 3^{2} \mid k$.

Step 7 . We have $k \equiv 322560$ or $1298592(\bmod 1952064)$. In the case $k \equiv$ $1298592(\bmod 1952064)$ we have $k \equiv 288(\bmod 576)$. The latter is impossible, since $(288,577)_{432}$ is a helpful pair. Hence $k \equiv 322560(\bmod 1952064)$ and $2^{6} \cdot 3^{2} \mid k$.

Step 8 . We have $k \equiv 322560$ or 2274624 or $4226688(\bmod 5856192)$. The case $k \equiv 322560(\bmod 5856192)$ is impossible, since in this case $k \equiv 288(\bmod 432)$ and $(288,433)_{284}$ is a helpful pair. In the case $k \equiv 2274624(\bmod 5856192)$ we have $k \equiv 36(\bmod 108)$, which is impossible as $(36,109)_{21}$ is a helpful pair. Hence $k \equiv 4226688(\bmod 5856192)$ and $2^{6} \cdot 3^{3} \mid k$.

Step 9 . We have $k \equiv 4226688$ or 10082880 or 15939072 or 21795264 or 27651456 $(\bmod 29280960)$. If $k \equiv 15939072$ or $27651456(\bmod 29280960)$, then $k \equiv 6$ or 12 $(\bmod 30)$, which is impossible as $(6,31)_{21}$ and $(12,31)_{21}$ are helpful pairs. In the case $k \equiv 21795264(\bmod 29280960)$ we have $k \equiv 24(\bmod 60)$, which is impossible 
since $(24,61)_{8}$ is a helpful pair. The case $k \equiv 4226688(\bmod 29280960)$ is also impossible, since in this case $k \equiv 108(\bmod 180)$ and $(108,181)_{82}$ is a helpful pair. Hence $k \equiv 10082880(\bmod 29280960)$ and $2^{6} \cdot 3^{3} \cdot 5 \mid k$.

Step 10. We have $k \equiv 10082880+29280960 j(\bmod 204966720)$ for some $j \in$ $\{0,1, \ldots, 6\}$. If $j \in\{0,1,3,6\}$ then $k \equiv 8$ or 12 or 16 or $24(\bmod 28)$, which is impossible as $(8,29)_{22},(12,29)_{22},(16,29)_{22}$ and $(24,29)_{22}$ are helpful pairs. If $j=4$ or 5 , then we have $k \equiv 6$ or $18(\bmod 42)$. The latter is impossible, since $(6,43)_{28}$ and $(18,43)_{28}$ are helpful pairs. Hence $k \equiv 68644800(\bmod 204966720)$ and $2^{6} \cdot 3^{3} \cdot 5 \cdot 7 \mid k$.

Step 11. We have $k \equiv 68644800+204966720 j(\bmod 1024833600)$ for some $j \in\{0,1,2,3,4\}$. If $j \in\{1,2,3\}$, then $k \equiv 20$ or 40 or $60(\bmod 100)$, which is impossible as $(20,101)_{12},(40,101)_{12}$ and $(60,101)_{12}$ are helpful pairs. The case $j=4$ is also impossible, since in this case $k \equiv 3780(\bmod 6300)$ and $(3780,6301)_{478}$ is a helpful pair. Hence $k \equiv 68644800(\bmod 1024833600)$ and $2^{6} \cdot 3^{3} \cdot 5^{2} \cdot 7 \mid k$.

Step 12. We have $k \equiv 68644800+1024833600 j(\bmod 11273169600)$ for some $j \in\{0,1, \ldots, 10\}$. If $j \in\{0,2,3,6,8\}$, then $k \equiv 2$ or 4 or 12 or 18 or $20(\bmod 22)$, which is impossible as $(2,23)_{17},(4,23)_{17},(12,23)_{17},(18,23)_{17}$ and $(20,23)_{17}$ are helpful pairs. If $j \in\{1,5,7\}$, then $k \equiv 30$ or 36 or 54 ( $\bmod 66)$, which is impossible since $(30,67)_{12},(36,67)_{12}$ and $(54,67)_{12}$ are helpful pairs. In the case $j=10$ we have $k \equiv 16(\bmod 88)$, which is impossible as $(16,89)_{15}$ is a helpful pair. The case $j=9$ is also impossible, since in this case $k \equiv 160(\bmod 352)$ and $(160,353)_{72}$ is a helpful pair. Hence $k \equiv 4167979200(\bmod 11273169600)$ and $2^{6} \cdot 3^{3} \cdot 5^{2} \cdot 7 \cdot 11 \mid k$.

Step 13 . We have $k \equiv 4167979200+11273169600 j(\bmod 146551204800)$ for some $j \in\{0,1, \ldots, 12\}$. If $j \in\{1,3,4,5,7,8,10\}$, then $k \equiv 12$ or 20 or 24 or 28 or 36 or 40 or $48(\bmod 52)$, which is impossible as $(12,53)_{48},(20,53)_{48},(24,53)_{48},(28,53)_{48}$, $(36,53)_{48},(40,53)_{48}$ and $(48,53)_{48}$ are helpful pairs. If $j=2$ or 12 , then $k \equiv$ 30 or $42(\bmod 78)$, which is impossible, since $(30,79)_{64}$ and $(42,79)_{64}$ are helpful pairs. If $j=0$ or 6 , then $k \equiv 60$ or $110(\bmod 130)$, which is impossible as $(60,131)_{98}$ and $(110,131)_{98}$ are helpful pairs. The case $j=9$ is also impossible, since in this case $k \equiv 96(\bmod 156)$ and $(96,157)_{28}$ is a helpful pair. Hence $k \equiv 128172844800$ $(\bmod 146551204800)$ and $2^{6} \cdot 3^{3} \cdot 5^{2} \cdot 7 \cdot 11 \cdot 13 \mid k$.

It seems that the type of argument used in the proof of Proposition 7 can be continued to deduce that more and more small prime factors must divide $k$. Given a prime $q \geq 5$ and $2 \leq t \leq q-3$ even, one would heuristically expect that $(t, q)_{a}$ is helpful with probability $(1-1 / q)^{q-1}$ which tends to $1 / e$, on assuming that the values $S_{t}(c)$ are randomly distributed modulo $q$. The numerical data obtained so far turn out to be consistent with this.

For the original Erdős-Moser equation it is known (cf. [9, 21]) that $N \mid k$ with

$$
N=2^{8} \cdot 3^{5} \cdot 5^{4} \cdot 7^{3} \cdot 11^{2} \cdot 13^{2} \cdot 17^{2} \cdot 19^{2} \cdot 23 \cdots 997>5.7462 \cdot 10^{427} .
$$

An heuristic argument can be given suggesting that if, say $L_{v}:=\operatorname{lcm}(1,2, \ldots, v)$ divides $k$, with tremendously high likelihood we can infer that $L_{w}$ divides $k$, where $w$ is the smallest prime not dividing $L_{v}$. It is already enough to have $v \geq 11$ here. To deduce that $k$ is divisible by say 24 might be delicate, but once one has $L_{v} \mid k$ say, 
there is an explosion of further helpful pairs one can use to establish divisibility of $k$ by an even larger integer. To add the first prime $w$ not dividing $L_{v}$, one needs to have only a number of helpful pairs that is roughly linear in $v$, whereas an exponential number (in $v$ ) is available. However, the required computation time goes sharply up with increasing $w$.

This result gives a lower bound of $10^{427}$ for $k$, which is modest in comparison with the lower bound obtained by Moser. However, as argued by Gallot et al. [7], a result of the form $N \mid k$ leads to an expected lower bound $m>10^{257 N}$. For the Kellner-Erdős-Moser equation we likewise expect a result of the form $N \mid k$ to lead to a lower bound for $m$ that is exponential in $N$.

Unfortunately, the authors are not aware of any systematic approach that would allow one to prove a result of the type that if $a S_{k}(m)=m^{k}$, then $120 \mid k$, for every $a \geq 1$. Some preliminary work on this for the equation $S_{k}(m)=a m^{k}$ was done by the second author's intern Muriel Lang [13] in 2009.

\section{LOWER BOUND FOR $m$}

The aim of this section is to establish Theorem 7 . The proof rests on Lemmas 12 and 13 .

Lemma 12. Suppose that $a S_{k}(m)=m^{k}$ with $m \geq 4$ and $k$ even. Then $m-1$ and $2 m-1$ are square-free, and if $p$ is a prime divisor of $(m-1)(2 m-1)$, then $(p-1) \mid k$.

Proof. Since

$$
S_{k}(m-1)=S_{k}(m)-(m-1)^{k} \equiv S_{k}(m) \quad(\bmod m-1),
$$

Lemma 1 yields

$$
a \sum_{\substack{p|(m-1) \\(p-1)| k}} \frac{m-1}{p}+m^{k} \equiv 0 \quad(\bmod a(m-1))
$$

Note that if $p \mid(m-1)$ and $(p-1) \nmid k$, then $p \mid m$, a contradiction that shows that $p \mid(m-1)$ implies $(p-1) \nmid k$. If $p^{2} \mid(m-1)$ it follows again that $p \mid m$, a contradiction that shows that $m-1$ is square-free.

Note that

$$
S_{k}(2 m-1)=\sum_{j=1}^{m-1}\left(j^{k}+(2 m-1-j)^{k}\right) \equiv 2 S_{k}(m) \quad(\bmod 2 m-1) .
$$

Then, again by Lemma 1,

$$
a \sum_{\substack{p|(2 m-1) \\(p-1)| k}} \frac{2 m-1}{p}+2 m^{k} \equiv 0 \quad(\bmod a(2 m-1)),
$$

from which we deduce that $2 m-1$ is square-free and each prime $p$ dividing $2 m-1$ satisfies $(p-1) \mid k$.

Corollary 9. Suppose that $a S_{k}(m)=m^{k}$ with $m \geq 4$ and $k$ even, then $m \equiv 3$ $(\bmod 4)$. 
Lemma 13. Suppose that $a S_{k}(m)=m^{k}$ with $m \geq 4$ and $k$ even and let $p$ be a prime divisor of $(m+1)(2 m+1)$. If $(p-1) \mid k$ then $\operatorname{ord}_{p}((m+1)(2 m+1))=\operatorname{ord}_{p}(a+1)+1$, otherwise $\operatorname{ord}_{p}((m+1)(2 m+1)) \leq \operatorname{ord}_{p}(a+1)$.

Proof. Observe that $a S_{k}(m+1)=(a+1) m^{k}$. Invoking Lemma 1, we obtain

$$
a \sum_{\substack{p|(m+1) \\(p-1)| k}} \frac{m+1}{p}+(a+1) m^{k} \equiv 0 \quad(\bmod a(m+1)) .
$$

Since $p \mid a$ implies $p \mid m$ it follows that $\operatorname{gcd}(a, m+1)=1$. Thus

$$
\begin{array}{ll}
\operatorname{ord}_{p}(m+1)=\operatorname{ord}_{p}(a+1)+1 & \text { if } p \mid(m+1) \text { and }(p-1) \mid k, \\
\operatorname{ord}_{p}(m+1) \leq \operatorname{ord}_{p}(a+1) & \text { if } p \mid(m+1) \text { and }(p-1) \nmid k .
\end{array}
$$

Further, from

$a S_{k}(2 m+1)=a \sum_{j=1}^{m}\left(j^{k}+(2 m+1-j)^{k}\right) \equiv 2 a S_{k}(m+1) \equiv 2(a+1) m^{k} \quad(\bmod a(2 m+1))$ we deduce that

$$
a \sum_{\substack{p|(2 m+1) \\(p-1)| k}} \frac{2 m+1}{p}+2(a+1) m^{k} \equiv 0 \quad(\bmod a(2 m+1))
$$

and so

$$
\begin{array}{ll}
\operatorname{ord}_{p}(2 m+1)=\operatorname{ord}_{p}(a+1)+1 & \text { if } p \mid(2 m+1) \text { and }(p-1) \mid k, \\
\operatorname{ord}_{p}(2 m+1) \leq \operatorname{ord}_{p}(a+1) & \text { if } p \mid(2 m+1) \text { and }(p-1) \nmid k .
\end{array}
$$

Since $m+1$ and $2 m+1$ are coprime, the asserted result follows.

Part (g) below arose in collaboration with Jan Büthe (University of Bonn) and we only provide a sketch of the proof here. In a planned sequel to this paper [4] further details will be given. We remark that if the condition $m \equiv 1(\bmod 30)$ is replaced by

$$
\sum_{p \mid(m-1)} \frac{1}{p}+\frac{1}{a}>1
$$

(cf. equation (22)) the same conclusion holds true.

Theorem 7. Assume that $a>1$ is square-free and that $a S_{k}(m)=m^{k}$ with $m \geq 4$ and $k$ even. Put $a_{1}=\operatorname{gcd}(a+1, m+1)$ and $a_{2}=\operatorname{gcd}(a+1,2 m+1)$. Put

$$
M=\frac{\left(m^{2}-1\right)\left(4 m^{2}-1\right)}{6 a_{1} a_{2}} .
$$

Then

(a) $m>a$;

(b) $m-1,2 m-1,(m+1) / a_{1}$, and $(2 m+1) / a_{2}$ are all square-free;

(c) if $p$ divides at least one of the above four integers, then $(p-1) \mid k$;

(d) $m>3.4429 \cdot 10^{82}$;

(e) the number $M$ is square-free and has at least 139 prime factors; 
(f) if $m \equiv 1(\bmod 3)$, then $m>1.485 \cdot 10^{9321155}$ and the number $M$ has at least 4990906 prime factors;

(g) if $m \equiv 1(\bmod 30)$, then $m>10^{4 \cdot 10^{20}}$ and the number $M$ has at least 75760524354901799895 prime factors.

Proof. As $a$ is square-free, we have $a \mid m$, and so $m \geq a$. If $m=a$, then (14) yields

$$
\sum_{\substack{p|(m+1) \\(p-1)| k}} \frac{1}{p} \equiv 0 \quad(\bmod 1)
$$

Since the sum of reciprocals of distinct primes can never be a positive integer, we must have

$$
\sum_{\substack{p|(m+1) \\(p-1)| k}} \frac{1}{p}=0
$$

which contradicts the fact that $2 \mid(m+1)$. Parts $(\mathrm{b})$ and $(\mathrm{c})$ are direct consequences of Lemmas 12 and 13. Further, using Lemma 13, parts (b) and (c) and the facts that $a \mid m$ and $\operatorname{gcd}(a, m-1)=\operatorname{gcd}(a, 2 m-1)=\operatorname{gcd}(a, m+1)=\operatorname{gcd}(a, 2 m+1)=1$, we find that

$$
\begin{aligned}
m^{k} & \equiv m \quad(\bmod a(m-1)), \\
2 m^{k} & \equiv 4 m \quad(\bmod a(2 m-1)), \\
(a+1) m^{k} & \equiv a+1+(a-1)(m+1) \quad(\bmod a(m+1)), \\
2(a+1) m^{k} & \equiv 2(a+1)+(a-2)(2 m+1) \quad(\bmod a(2 m+1)) .
\end{aligned}
$$

Here we will only provide details for the latter congruence, which is the most complicated one to establish. Since $a$ and $2 m+1$ are coprime, it suffices by the Chinese remainder theorem to establish the congruence modulo $a$ and modulo $2 m+1$. Since $a \mid m$ the congruence trivially holds modulo $a$.

Now suppose that $p \mid(2 m+1)$.

First case: $(p-1) \mid k$.

By Lemma 13 we have $\operatorname{ord}_{p}(2 m+1)=\operatorname{ord}_{p}(a+1)+1$ and it suffices to show that $m^{k} \equiv 1(\bmod p)$. This is true by Euler's theorem.

Second case: $(p-1) \nmid k$.

Here we use that, by Lemma 13 again, $\operatorname{ord}_{p}(2 m+1) \leq \operatorname{ord}_{p}(a+1)$ to see that the congruence holds. 
We can rewrite the congruences (12) - (15) as

$$
\begin{aligned}
\sum_{p \mid(m-1)} \frac{1}{p}+\frac{m}{a(m-1)} & \equiv 0 \quad(\bmod 1), \\
\sum_{p \mid(2 m-1)} \frac{1}{p}+\frac{4 m}{a(2 m-1)} & \equiv 0 \quad(\bmod 1), \\
\sum_{p \mid \frac{m+1}{a_{1}}} \frac{1}{p}+\frac{a+1+(a-1)(m+1)}{a(m+1)} & \equiv 0 \quad(\bmod 1), \\
\sum_{p \mid \frac{2 m+1}{a_{2}}} \frac{1}{p}+\frac{2(a+1)+(a-2)(2 m+1)}{a(2 m+1)} & \equiv 0 \quad(\bmod 1) .
\end{aligned}
$$

By Corollary 9, the assumption that $k$ is even and Lemma 13, we see that $(m+1) / a_{1}$ is even. Now noting that $a \geq 37$ (by Corollary [6] and the fact that 37 is the first irregular prime), we have

$$
\sum_{p \mid \frac{m+1}{a_{1}}} \frac{1}{p}+\frac{a+1+(a-1)(m+1)}{a(m+1)} \geq \frac{1}{2}+\frac{a+1+a(m+1)}{a(m+1)}-\frac{1}{a}>1 .
$$

Therefore, if we add the left hand sides of (16), (17), (18) and (19), we get an integer, at least 5 . No prime $p>3$ can divide more than one of the integers $m-1,2 m-1$, $(m+1) / a_{1}$, and $(2 m+1) / a_{2}$, and 2 and 3 divide precisely two of these integers. Hence $M=\left(m^{2}-1\right)\left(4 m^{2}-1\right) /\left(6 a_{1} a_{2}\right)$ is square-free and

$$
\begin{aligned}
\sum_{p \mid M} \frac{1}{p}+\frac{1}{a(m-1)} & +\frac{2}{a(2 m-1)}+\frac{a+1}{a(m+1)}+\frac{2(a+1)}{a(2 m+1)} \\
& \geq 3-\frac{1}{2}-\frac{1}{3}=2 \frac{1}{6} .
\end{aligned}
$$

Since $a \mid m, m>a \geq 37$ and each prime divisor of $m$ is irregular, we have $m \geq 37^{2}$. A simple computation shows that (16) is never satisfied for $a \geq 37$ and $m=37^{2}$. Since 59 is the second irregular prime, it follows that $m \geq 37 \cdot 59$. On noting that the four fractions above are decreasing functions in both $a$ and $m$, we find on substituting $a=37$ and $m=37 \cdot 59$ that $\sum_{p \mid M} \frac{1}{p}>\alpha$, with $\alpha=2.1657$. Note that if

$$
\sum_{p \leq x} \frac{1}{p}<\alpha
$$

then $m^{4} / 3>M>\prod_{p \leq x} p$ (note that $a_{1} \geq 2$ and hence $M<m^{4} / 3$ ). One computes (using a computer algebra package) the largest prime $p_{s}$ such that $\sum_{p_{j} \leq p_{s}} \frac{1}{p_{j}}<2 \frac{1}{6}$, with $p_{1}, p_{2}, \ldots$ the consecutive primes. Here one finds that $s=139$ and

$$
\sum_{j=1}^{139} \frac{1}{p_{j}}<2.16566<\alpha<\sum_{p \mid M} \frac{1}{p} .
$$


Thus

$$
m>\left(3 \prod_{p \leq p_{139}} p\right)^{1 / 4}>3.4429 \cdot 10^{82}
$$

Now assume that $m \equiv 1(\bmod 3)$. Then $3 \mid(2 m+1) / a_{1}$ by Lemma 13 , and so

$$
\sum_{p \mid \frac{2 m+1}{a_{2}}} \frac{1}{p}+\frac{2(a+1)+(a-2)(2 m+1)}{a(2 m+1)} \geq \frac{1}{3}+\frac{2(a+1)+a(2 m+1)}{a(2 m+1)}-\frac{1}{a / 2}>1 .
$$

Hence in this case, $2 \frac{1}{6}$ in (20) can be replaced by $3 \frac{1}{6}$. This $\alpha$ occurs in the work of Moser and here it is known that $s=4990906$ leading to $m>1.485 \cdot 10^{9321155}$ (cf. [5, 18]).

Finally, assume that $m \equiv 1(\bmod 30)$. Then

$$
\sum_{p \mid(m-1)} \frac{1}{p}+\frac{m}{a(m-1)}>\frac{1}{2}+\frac{1}{3}+\frac{1}{5}>1
$$

and we have the inequality (20) with $2 \frac{1}{6}$ replaced by $4 \frac{1}{6}$. In this case the largest prime $p_{s}$ such that $\sum_{p \leq p_{s}} \frac{1}{p}<4 \frac{1}{6}$ can no longer be determined by direct computation and more sophisticated methods are needed, cf. [2, 4].

Remark. Note that in the proof we only used that $a \geq 37$. This has as a consequence that the proof only depends on the first assertion in Theorem 4

\section{Proofs of the NeW Results Announced in the introduction}

It remains to establish Theorem 4, Theorem 5 and Proposition 1.

Proof of Theorem 4. The first restriction on $a$ arises on invoking Corollary 6. In order to obtain the second restriction we have two write down all integers $a \leq 1500$ that are composed only of irregular primes. These are listed in Table 3, Each of these can be excluded as is shown for two examples in Section 5. This exclusion process for each a can be reconstructed using Table 3 .

To prove Theorem 5 we need the following result, which shows that $m$ and $k$ are of comparable size.

Lemma 14. Suppose that $a S_{k}(m)=m^{k}$. Then $k+1<a m<(a+1)(k+1)$.

Proof. We have

$$
S_{k}(m) \leq \int_{1}^{m} x^{k} \mathrm{~d} x \leq S_{k}(m+1)-1
$$

Hence

$$
m^{k}=a S_{k}(m) \leq a \int_{1}^{m} x^{k} \mathrm{~d} x=\frac{a\left(m^{k+1}-1\right)}{k+1}<\frac{a m^{k+1}}{k+1}
$$


and so $a m>k+1$. Further,

$$
(a+1) m^{k}=a S_{k}(m+1) \geq a\left(1+\int_{1}^{m} x^{k} \mathrm{~d} x\right)=\frac{a\left(m^{k+1}+k\right)}{k+1}>\frac{a m^{k+1}}{k+1},
$$

that is $a m<(a+1)(k+1)$.

Proof of Theorem 5. The lower bound on $m$ is a consequence of Theorem 7 , part (d). On invoking Lemma 14 with $a \geq 1501$ the result follows.

Proof of Proposition 1. Let $(m, k)$ be a solution of (3). Observe that

$$
\frac{S_{j}(m)}{m^{j}}=\left(\frac{1}{m}\right)^{j}+\left(\frac{2}{m}\right)^{j}+\cdots+\left(\frac{m-1}{m}\right)^{j}>\frac{S_{j+1}(m)}{m^{j+1}},
$$

and so

$$
\begin{array}{ll}
a S_{j}(m)-m^{j}>0 & \text { if } j<k, \\
a S_{j}(m)-m^{j}<0 & \text { if } j>k .
\end{array}
$$

This shows that for every $m$, there is at most one $k$.

Now assume that there exists a positive integer $n$ such that $a S_{k}(m+n)=(m+n)^{k}$. Then $k>1$. Since

$$
S_{k}(m+n)=S_{k}(n)+\sum_{j=0}^{m-1}(n+j)^{k}=S_{k}(n)+m n^{k}+\sum_{j=1}^{k}\left(\begin{array}{l}
k \\
j
\end{array}\right) n^{k-j} S_{j}(m),
$$

we have

$$
(m+n)^{k}=\sum_{j=0}^{k}\left(\begin{array}{l}
k \\
j
\end{array}\right) m^{j} n^{k-j}=a S_{k}(n)+a m n^{k}+a \sum_{j=1}^{k}\left(\begin{array}{l}
k \\
j
\end{array}\right) n^{k-j} S_{j}(m),
$$

or, equivalently,

$$
n^{k}=a S_{k}(n)+a m n^{k}+\sum_{j=1}^{k-1}\left(\begin{array}{l}
k \\
j
\end{array}\right) n^{k-j}\left(a S_{j}(m)-m^{j}\right) .
$$

In view of (23), the last equality cannot hold. Thus we see that, for every $k$, there is at most one $m$.

Remark. Using the same type of argument, we can prove the following: if $\left(m_{1}, k_{1}\right)$ and $\left(m_{2}, k_{2}\right)$ are two distinct solutions of $a S_{k}(m)=m^{k}$ then either $m_{1}>m_{2}, k_{1}>k_{2}$ or $m_{1}<m_{2}, k_{1}<k_{2}$.

\section{Other Properties of Kellner-Erdős-Moser solutions}

There are many restrictions known that a solution of the Erdős-Moser equation has to satisfy. We expect that most of these have an analogue for the Kellner-ErdösMoser equation as well. We present an example. 
Theorem 8. Suppose that $a S_{k}(m)=m^{k}$ with $m \geq 4$ and $k$ even. We have

$$
\operatorname{ord}_{2}(a(m-1)-2) \begin{cases}=3+\operatorname{ord}_{2} k & \text { if } a \equiv 1 \quad(\bmod 4), \\ \geq 4+\operatorname{ord}_{2} k & \text { if } a \equiv 3 \quad(\bmod 4)\end{cases}
$$

For $a=1$ this was first established in Moree et al. [21, Lemma 12] using Bernoulli numbers. A much more elementary proof also dealing with solutions of the equation $S_{k}(m)=a m^{k}$ for integers $a$ was given later by the second author [17].

Our proof of Theorem 8 makes use of the following lemma.

Lemma 15. Let $k$ and $m$ be positive integers where $k \geq 6$ is even. Then

$$
S_{k}(m) \equiv\left\{\begin{array}{lll}
{\left[\frac{m}{2}\right]+2^{2+\operatorname{ord}_{2} k} \quad\left(\bmod 2^{3+\operatorname{ord}_{2} k}\right)} & \text { if }\left[\frac{m}{2}\right] \equiv 2 & (\bmod 4), \\
{\left[\frac{m}{2}\right] \quad\left(\bmod 2^{3+\operatorname{ord}_{2} k}\right)} & \text { otherwise. }
\end{array}\right.
$$

Proof. It is easily proved by induction on $s$ that for an odd integer $j$ and $s \geq 1$

$$
j^{2^{s}} \equiv\left\{\begin{array}{lll}
1 \quad\left(\bmod 2^{s+3}\right) & \text { if } j \equiv \pm 1 & (\bmod 8) \\
2^{s+2}+1 \quad\left(\bmod 2^{s+3}\right) & \text { if } j \equiv \pm 3 & (\bmod 8)
\end{array}\right.
$$

Note that $k \geq 3+\operatorname{ord}_{2} k$. Indeed, for $\operatorname{ord}_{2} k=1$ and $\operatorname{ord}_{2} k=2$ it follows from the condition $k \geq 6$ and for $\operatorname{ord}_{2} k \geq 3$ we have $k \geq 2^{\operatorname{ord}_{2} k} \geq 3+\operatorname{ord}_{2} k$. Thus for an integer $j$ we have

$$
j^{k} \equiv \begin{cases}0 \quad\left(\bmod 2^{3+\operatorname{ord}_{2} k}\right) & \text { if } j \text { is even } \\ 1 \quad\left(\bmod 2^{3+\operatorname{ord}_{2} k}\right) & \text { if } j \equiv \pm 1 \quad(\bmod 8) \\ 2^{2+\operatorname{ord}_{2} k}+1 \quad\left(\bmod 2^{3+\operatorname{ord}_{2} k}\right) & \text { if } j \equiv \pm 3 \quad(\bmod 8)\end{cases}
$$

Assume that $m \equiv 1(\bmod 4)$. Then

$\#\{1 \leq n<m: n \equiv \pm 1 \quad(\bmod 8)\}=\#\{1 \leq n<m: n \equiv \pm 3 \quad(\bmod 8)\}=\frac{m-1}{4}$.

Hence

$$
\begin{aligned}
& S_{k}(m) \equiv \frac{m-1}{4}+\frac{m-1}{4}\left(2^{2+\operatorname{ord}_{2} k}+1\right) \equiv \frac{m-1}{2}\left(2^{1+\operatorname{ord}_{2} k}+1\right) \\
& \equiv\left\{\begin{array}{lll}
\frac{m-1}{2}\left(\bmod 2^{3+\operatorname{ord}_{2} k}\right) & \text { if } m \equiv 1 & (\bmod 8), \\
\frac{m-1}{2}+2^{2+\operatorname{ord}_{2} k} \quad\left(\bmod 2^{3+\operatorname{ord}_{2} k}\right) & \text { if } m \equiv 5 & (\bmod 8) .
\end{array}\right.
\end{aligned}
$$

Now assume that $m \equiv 3(\bmod 4)$. Then

$\#\{1 \leq n \leq m: n \equiv \pm 1(\bmod 8)\}=\#\{1 \leq n \leq m: n \equiv \pm 3 \quad(\bmod 8)\}=\frac{m+1}{4}$.

This yields

$$
\begin{aligned}
& S_{k}(m+1) \equiv \frac{m+1}{4}+\frac{m+1}{4}\left(2^{2+\operatorname{ord}_{2} k}+1\right) \equiv \frac{m+1}{2}\left(2^{1+\operatorname{ord}_{2} k}+1\right)
\end{aligned}
$$

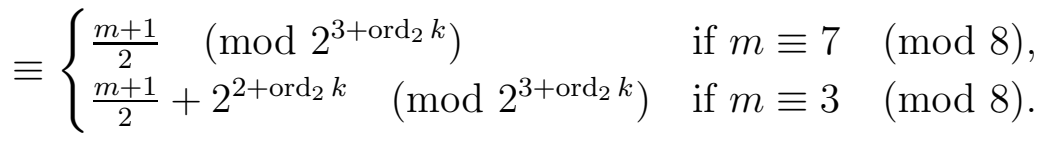


Since

$$
m^{k} \equiv\left\{\begin{array}{lll}
1 \quad\left(\bmod 2^{3+\operatorname{ord}_{2} k}\right) & \text { if } m \equiv 7 \quad(\bmod 8) \\
2^{2+\operatorname{ord}_{2} k}+1 \quad\left(\bmod 2^{3+\operatorname{ord}_{2} k}\right) & \text { if } m \equiv 3 \quad(\bmod 8)
\end{array}\right.
$$

we deduce that

$$
S_{k}(m)=S_{k}(m+1)-m^{k} \equiv \frac{m-1}{2} \quad\left(\bmod 2^{3+\operatorname{ord}_{2} k}\right) .
$$

Finally, if $m$ is even then

$$
S_{k}(m) \equiv S_{k}(m+1) \equiv\left\{\begin{array}{lll}
\frac{m}{2}\left(\bmod 2^{3+\operatorname{ord}_{2} k}\right) & \text { if } m \neq \equiv 4 & (\bmod 8) \\
\frac{m}{2}+2^{2+\operatorname{ord}_{2} k}\left(\bmod 2^{3+\operatorname{ord}_{2} k}\right) & \text { if } m \equiv 4 & (\bmod 8)
\end{array}\right.
$$

This completes the proof.

Proof of Theorem 8, By Lemma [15] and Corollaries 4 and 9 ,

$$
S_{k}(m) \equiv \frac{m-1}{2} \quad\left(\bmod 2^{3+\operatorname{ord}_{2} k}\right) .
$$

From Lemma 13 we see that

$$
m \equiv\left\{\begin{array}{lll}
7 & (\bmod 8) & \text { if } a \equiv 3 \quad(\bmod 4) \\
3 & (\bmod 8) & \text { if } a \equiv 1 \quad(\bmod 4)
\end{array}\right.
$$

Therefore

$$
a S_{k}(m) \equiv a \cdot \frac{m-1}{2} \equiv m^{k} \equiv \begin{cases}1 \quad\left(\bmod 2^{3+\operatorname{ord}_{2} k}\right) & \text { if } a \equiv 3 \quad(\bmod 4), \\ 2^{2+\operatorname{ord}_{2} k}+1 \quad\left(\bmod 2^{3+\operatorname{ord}_{2} k}\right) & \text { if } a \equiv 1 \quad(\bmod 4)\end{cases}
$$

and so

$$
a(m-1)-2 \equiv\left\{\begin{array}{lll}
0 \quad\left(\bmod 2^{4+\operatorname{ord}_{2} k}\right) & \text { if } a \equiv 3 \quad(\bmod 4) \\
2^{3+\operatorname{ord}_{2} k}\left(\bmod 2^{4+\operatorname{ord}_{2} k}\right) & \text { if } a \equiv 1 \quad(\bmod 4)
\end{array}\right.
$$

as desired.

Theorem 9. Let $a>3$. If $a S_{k}(m)=m^{k}$ and $m$ is a prime, then $a=q^{2 s}$ for some irregular prime $q \equiv 3(\bmod 16)$ and positive integer $s$.

Proof. If $a$ has at least two distinct prime divisors, then $m$ cannot be a prime. Now assume that $a$ is a power of a prime $q$. Then $q$ is an irregular prime and $m=q$. Suppose that $a=q^{2 s+1}$ for some $s \geq 0$. Then $(a+1) m^{k} / a(m+1)=$ $\left(q^{2 s+1}+1\right) S_{k}(q) /(q+1)$ is an integer, and (14) implies

$$
\sum_{\substack{p|(q+1) \\(p-1)| k}} \frac{1}{p} \equiv 0 \quad(\bmod 1) .
$$

Exactly as in the proof of Theorem 7 , we conclude that this is impossible. Finally, assume that $a=q^{2 s}$ for some $s \geq 1$. Then $a \equiv 1(\bmod 8)$. Note that $k$ has to be even. By Theorem 8 it follows that $a(q-1) \equiv 2(\bmod 16)$, which yields $\frac{q-1}{2} \equiv 1$ $(\bmod 8)$, that is, $q \equiv 3(\bmod 16)$. 
Remark. It is not known whether there are infinitely many irregular primes $q \equiv 3$ $(\bmod 16)$. However, from a result of Metsänkylä [15] it follows that there are infinitely many irregular primes $q \equiv \pm 3, \pm 5(\bmod 16)$.

Acknowledgement. This paper was begun during the stay of the first author in February-April 2014 at the Max Planck Institute for Mathematics. She likes to thank for the invitation and the pleasant research atmosphere. The second author was introduced to the subject around 1990 by the late Jerzy Urbanowicz. He will never forget his interest, help and kindness. Further he thanks Prof. T. N. Shorey for helpful discussions in the summer of 2014. We like to heartily thank Bernd Kellner for helpful e-mail correspondence and patiently answering our Bernoulli number questions. A. Ciolan, P. Tegelaar and W. Zudilin kindly commented on an earlier version of this paper.

The cooperation of the authors has its origin in them having met, in 2012, at ELAZ in Schloss Schney (which was also attended by Prof. W. Schwarz). This contribution is our tribute to Prof. W. Schwarz, who was one of the initiators of the ELAZ conference series.

\section{APPENDIX}

Table 1: Pairs of irregular primes $\left(p_{1}, p_{2}\right)$ with $p_{1}<p_{2}$, $p_{1} p_{2}<50000$, satisfying the conditions of Corollary 7

\begin{tabular}{|c|c|}
\hline$p_{1}$ & $p_{2}$ \\
\hline 37 & $\begin{array}{l}67,103,149,157,271,307,379,401,409,421,433,463,523,541,547, \\
557,577,593,607,613,631,673,727,757,811,877,881,1061,1117 \\
1129,1153,1193,1201,1237,1297,1327\end{array}$ \\
\hline 59 & 233,523 \\
\hline 67 & $\begin{array}{l}103,157,271,283,409,421,433,463,541,547,613,617,619,683,691 \text {, } \\
727\end{array}$ \\
\hline 101 & $131,149,157,271,311,401,409,421,433,461$ \\
\hline 103 & $157,283,307,463$ \\
\hline 131 & 157,271 \\
\hline 149 & $233,257,293$ \\
\hline 157 & $233,257,271,293,307$ \\
\hline
\end{tabular}

Table 2: Helpful pairs $(t, q)_{a}$ with $q \leq 17$

\begin{tabular}{|c|c|l|}
\hline$q$ & $t$ & \multicolumn{1}{|c|}{$a(\bmod q)$} \\
\hline 5 & 2 & $1,2,3$ \\
\hline 7 & 2 & $1,3,5$ \\
\hline 7 & 4 & $1,3,5$ \\
\hline 11 & 2 & $1,2,3,5,6,7$ \\
\hline
\end{tabular}


Table 2: Helpful pairs $(t, q)_{a}$ with $q \leq 17$

\begin{tabular}{|c|c|l|}
\hline$q$ & $t$ & \multicolumn{1}{|c|}{$a(\bmod q)$} \\
\hline 11 & 4 & $2,3,6,7,9$ \\
\hline 11 & 6 & $1,5,6,7$ \\
\hline 11 & 8 & $6,7,9$ \\
\hline 13 & 2 & $1,2,5,6,8,10,11$ \\
\hline 13 & 4 & $1,2,6,8,11$ \\
\hline 13 & 6 & $2,3,4,5,7,8,9,10,11$ \\
\hline 13 & 8 & $1,2,6,8,11$ \\
\hline 13 & 10 & $1,2,4,6,7,8,11$ \\
\hline 17 & 2 & $1,2,5,7,8,10,13,14$ \\
\hline 17 & 4 & $1,2,3,5,8,9,11,12,15$ \\
\hline 17 & 6 & $1,2,4,5,6,8$ \\
\hline 17 & 8 & $2,3,4,5,6,7,9,10,11,12,13,14,15$ \\
\hline 17 & 10 & $3,7,9,11,12,14,15$ \\
\hline 17 & 12 & $1,2,3,5,7,8,12,14,15$ \\
\hline 17 & 14 & $3,4,6,7,9,11,12,14,15$ \\
\hline
\end{tabular}

Table 3: Irregular pairs $(r, p)$ along with the corresponding helpful pairs $\left(t_{j}, q_{j}\right)_{a\left(\bmod q_{j}\right)}$ satisfying the conditions of Proposition 4

\begin{tabular}{|c|c|c|c|}
\hline$a$ & irregular pair $(r, p)$ & $\ell_{r}$ & helpful pairs $\left(t_{j}, q_{j}\right)_{a\left(\bmod q_{j}\right)}$ \\
\hline 37 & $(32,37)$ & 1 & $(8,13)_{11}$ \\
\hline 59 & $(44,59)$ & 6 & $(2,7)_{3},(4,7)_{3},(6,13)_{7},(276,349)_{59}$ \\
\hline 67 & $(58,67)$ & 2 & $(4,13)_{2},(10,13)_{2}$ \\
\hline 101 & $(68,101)$ & 6 & $(2,7)_{3},(4,7)_{3},(28,41)_{19},(168,601)_{101}$ \\
\hline 103 & $(24,103)$ & 14 & $\begin{array}{l}(2,5)_{3},(16,29)_{16},(24,43)_{17},(92,239)_{103}, \\
(194,239)_{103},(228,239)_{103},(636,1429)_{103}, \\
(840,1429)_{103}\end{array}$ \\
\hline 131 & $(22,131)$ & 4 & $(2,5)_{1},(12,41)_{8},(152,521)_{131}$ \\
\hline 149 & $(130,149)$ & 15 & $\begin{array}{l}(2,11)_{6},(4,11)_{6},(6,11)_{6},(8,11)_{6},(2,13)_{6}, \\
(10,13)_{6},(30,61)_{27}\end{array}$ \\
\hline 157 & $\begin{array}{l}(62,157) \\
(110,157)\end{array}$ & $\begin{array}{l}1 \\
1\end{array}$ & $\begin{array}{l}(2,5)_{2} \\
(2,5)_{2}\end{array}$ \\
\hline 233 & $(84,233)$ & 1 & $(26,59)_{56}$ \\
\hline 257 & $(164,257)$ & 1 & $(4,17)_{2}$ \\
\hline 263 & $(100,263)$ & 30 & $\begin{array}{l}(2,5)_{3},(2,31)_{15},(4,31)_{15},(8,31)_{15},(14,31)_{15} \\
(16,31)_{15},(22,31)_{15},(28,31)_{15},(40,61)_{19} \\
(624,787)_{263},(2196,2621)_{263},(2720,3931)_{263}\end{array}$ \\
\hline 271 & $(84,271)$ & 1 & $(4,11)_{7}$ \\
\hline
\end{tabular}


Table 3: Irregular pairs $(r, p)$ along with the corresponding helpful pairs $\left(t_{j}, q_{j}\right)_{a\left(\bmod q_{j}\right)}$ satisfying the conditions of Proposition 4

\begin{tabular}{|c|c|c|c|}
\hline$a$ & irregular pair $(r, p)$ & $\ell_{r}$ & helpful pairs $\left(t_{j}, q_{j}\right)_{a\left(\bmod q_{j}\right)}$ \\
\hline 283 & $(20,283)$ & 1 & $(2,7)_{3}$ \\
\hline 293 & $(156,293)$ & 30 & $\begin{array}{l}(2,11)_{7},(4,11)_{7},(6,11)_{7},(8,11)_{7},(20,41)_{6}, \\
(40,61)_{49},(740,877)_{293},(1032,1753)_{293}\end{array}$ \\
\hline 307 & $(88,307)$ & 1 & $(88,103)_{101}$ \\
\hline 311 & $(292,311)$ & 1 & $(2,11)_{3}$ \\
\hline 347 & $(280,347)$ & 30 & $\begin{array}{l}(2,5)_{2},(2,11)_{6},(4,11)_{6},(6,11)_{6},(8,11)_{6}, \\
(10,31)_{6},(20,61)_{42},(972,1039)_{347}\end{array}$ \\
\hline 353 & $\begin{array}{l}(186,353) \\
(300,353)\end{array}$ & $\begin{array}{l}1 \\
9\end{array}$ & $\begin{array}{l}(2,5)_{3} \\
(2,7)_{3},(4,7)_{3},(12,37)_{20},(24,37)_{20},(36,73)_{61}\end{array}$ \\
\hline 379 & $\begin{array}{l}(100,379) \\
(174,379)\end{array}$ & $\begin{array}{l}1 \\
1\end{array}$ & $\begin{array}{l}(4,7)_{1} \\
(48,127)_{99}\end{array}$ \\
\hline 389 & $\overline{(200,389)}$ & 4 & $(4,17)_{15},(8,17)_{15},(12,17)_{15},(976,1553)_{389}$ \\
\hline 401 & $(382,401)$ & 1 & $(2,5)_{1}$ \\
\hline 409 & $(126,409)$ & 3 & $(6,19)_{10},(12,19)_{10},(18,37)_{2}$ \\
\hline 421 & $(240,421)$ & 2 & $(20,41)_{11},(240,281)_{140}$ \\
\hline 433 & $(366,433)$ & 1 & $(2,5)_{3}$ \\
\hline 461 & $(196,461)$ & 1 & $(12,47)_{38}$ \\
\hline 463 & $(130,463)$ & 1 & $(4,7)_{1}$ \\
\hline 467 & $\begin{array}{l}(94,467) \\
(194,467)\end{array}$ & $\begin{array}{c}3 \\
18\end{array}$ & $\begin{array}{l}(2,7)_{5},(4,7)_{5},(1026,1399)_{467} \\
(2,5)_{2},(2,7)_{5},(4,7)_{5},(12,37)_{23},(24,37)_{23}, \\
(3456,8389)_{467}\end{array}$ \\
\hline 491 & $\begin{array}{l}(292,491) \\
(336,491) \\
(338,491) \\
\end{array}$ & $\begin{array}{l}1 \\
1 \\
1\end{array}$ & $\begin{array}{l}(2,11)_{7} \\
(6,11)_{7} \\
(8,11)_{7}\end{array}$ \\
\hline 523 & $(400,523)$ & 1 & $(4,7)_{5}$ \\
\hline 541 & $(86,541)$ & 1 & $(2,5)_{1}$ \\
\hline 547 & $\begin{array}{l}(270,547) \\
(486,547)\end{array}$ & $\begin{array}{l}1 \\
2\end{array}$ & $\begin{array}{l}(36,79)_{73} \\
(2,5)_{2},(96,157)_{76}\end{array}$ \\
\hline 557 & $(222,557)$ & 1 & $(2,5)_{2}$ \\
\hline 577 & $(52,577)$ & 1 & $(4,7)_{3}$ \\
\hline 587 & $\begin{array}{l}(90,587) \\
(92,587)\end{array}$ & 6 & $\begin{array}{l}(2,13)_{2},(4,13)_{2},(6,13)_{2},(8,13)_{2},(10,13)_{2}, \\
(90,1759)_{587} \\
(2,13)_{2},(4,13)_{2},(6,13)_{2},(8,13)_{2},(10,13)_{2}, \\
(678,1759)_{587}\end{array}$ \\
\hline 593 & $(22,593)$ & 1 & $(2,5)_{3}$ \\
\hline 607 & $(592,607)$ & 1 & $(4,7)_{5}$ \\
\hline 613 & $(522,613)$ & 1 & $(2,5)_{3}$ \\
\hline
\end{tabular}


Table 3: Irregular pairs $(r, p)$ along with the corresponding helpful pairs $\left(t_{j}, q_{j}\right)_{a\left(\bmod q_{j}\right)}$ satisfying the conditions of Proposition 4

\begin{tabular}{|c|c|c|l|}
\hline$a$ & irregular pair $(r, p)$ & $\ell_{r}$ & \multicolumn{1}{|c|}{ helpful pairs $\left(t_{j}, q_{j}\right)_{a\left(\bmod q_{j}\right)}$} \\
\hline 617 & $(20,617)$ & 1 & $(20,29)_{8}$ \\
& $(174,617)$ & 1 & $(2,5)_{2}$ \\
& $(338,617)$ & 1 & $(2,5)_{2}$ \\
\hline 619 & $(428,619)$ & 1 & $(2,7)_{3}$ \\
\hline 631 & $(80,631)$ & 1 & $(2,7)_{1}$ \\
& $(226,631)$ & 1 & $(4,7)_{1}$ \\
\hline 647 & $(236,647)$ & 6 & $(2,5)_{2},(2,7)_{3},(4,7)_{3},(84,229)_{189}$ \\
& $(242,647)$ & 3 & $(2,7)_{3},(4,7)_{3},(72,103)_{29}$ \\
& $(554,647)$ & 12 & $(2,5)_{2},(2,7)_{3},(4,7)_{3},(180,409)_{238}$, \\
& & & $(288,457)_{190}$ \\
\hline 653 & $(48,653)$ & 60 & $(8,17)_{7},(12,17)_{7},(4,31)_{2},(14,31)_{2},(12,41)_{38}$, \\
& & & $(20,41)_{38},(28,41)_{38},(24,61)_{43},(36,61)_{43}$, \\
& & & $(196,241)_{171},(48,2609)_{653},(9176,9781)_{653}$ \\
\hline 659 & $(224,659)$ & 18 & $(2,7)_{1},(4,7)_{1},(6,13)_{9},(12,19)_{13},(42,127)_{24}$, \\
& & & $(882,5923)_{659}$ \\
\hline 673 & $(408,673)$ & 1 & $(8,17)_{10}$ \\
& $(502,673)$ & 1 & $(2,5)_{3}$ \\
\hline 677 & $(628,677)$ & 3 & $(4,13)_{1},(8,13)_{1},(30,79)_{45}$ \\
\hline 683 & $(32,683)$ & 12 & $(2,5)_{3},(32,67)_{13},(76,89)_{60},(280,373)_{310}$, \\
& & & $(2760,4093)_{683}$ \\
\hline 691 & $(12,691)$ & 1 & $(12,31)_{9}$ \\
& $(200,691)$ & 1 & $(2,7)_{5}$ \\
\hline 727 & $(378,727)$ & 1 & $(4,23)_{14}$ \\
\hline 751 & $(290,751)$ & 1 & $(40,251)_{249}$ \\
\hline 757 & $(514,757)$ & 1 & $(2,5)_{2}$ \\
\hline 761 & $(260,761)$ & 1 & $(20,41)_{23}$ \\
\hline 773 & $(732,773)$ & 60 & $(2,7)_{3},(4,7)_{3},(2,11)_{3},(4,11)_{3},(4,17)_{8}$, \\
& & & $(12,17)_{8},(16,41)_{35},(120,241)_{50}$, \\
& & & $(1118,1931)_{773},(1504,3089)_{773}$ \\
\hline 797 & $(220,797)$ & 9 & $(8,37)_{20},(20,37)_{20},(220,2389)_{797}$, \\
& & & $(1812,2389)_{797},(2210,3583)_{797}$ \\
\hline 809 & $(330,809)$ & 5 & $(2,11)_{6},(4,11)_{6},(6,11)_{6},(8,11)_{6},(10,41)_{30}$ \\
& $(628,809)$ & 5 & $(2,11)_{6},(4,11)_{6},(6,11)_{6},(8,11)_{6},(20,41)_{30}$ \\
\hline 811 & $(544,811)$ & 1 & $(4,19)_{13}$ \\
\hline 821 & $(744,821)$ & 1 & $(4,11)_{7}$ \\
\hline 827 & $(102,827)$ & 6 & $(2,13)_{8},(4,13)_{8},(6,13)_{8},(8,13)_{8},(10,13)_{8}$, \\
& & & $(2580,4957)_{827}$ \\
\hline & & & \\
\hline
\end{tabular}


Table 3: Irregular pairs $(r, p)$ along with the corresponding helpful pairs $\left(t_{j}, q_{j}\right)_{a\left(\bmod q_{j}\right)}$ satisfying the conditions of Proposition 4

\begin{tabular}{|c|c|c|l|}
\hline$a$ & irregular pair $(r, p)$ & $\ell_{r}$ & \multicolumn{1}{|c|}{ helpful pairs $\left(t_{j}, q_{j}\right)_{a\left(\bmod q_{j}\right)}$} \\
\hline 839 & $(66,839)$ & 1680 & $(2,11)_{3},(4,11)_{3},(6,13)_{7},(10,13)_{7},(8,17)_{6}$, \\
& & & $(2,29)_{27},(4,29)_{27},(8,29)_{27},(10,29)_{27}$, \\
& & & $(12,29)_{27},(14,29)_{27},(16,29)_{27},(18,29)_{27}$, \\
& & & $(20,29)_{27},(22,29)_{27},(10,31)_{2},(20,31)_{2}$, \\
& & & $(10,41)_{19},(16,41)_{19},(20,41)_{19},(26,41)_{19}$, \\
& & & $(48,4)_{19},(30,41)_{19},(38,41)_{19},(28,61)_{46}$, \\
& & & $(4,97)_{63},(52,61)_{46},(28,71)_{58},(56,71)_{58}$, \\
& & & $(66,211)_{206},(6,281)_{277},(80,283)_{63},(26,211)_{206}$, \\
& & & $(138,281)_{277},(248,281)_{277},(258,281)_{277}$, \\
& & 1 & $(4,13)_{6}$ \\
\hline 877 & $(868,877)$ & 1 & $(2,5)_{1}$ \\
\hline 881 & $(162,881)$ & 3 & $(2,7)_{5},(4,7)_{5},(2190,2659)_{887}$ \\
\hline 887 & $(418,887)$ & & $(8,17)_{11}$ \\
\hline 929 & $(520,929)$ & 1 & $(4,17)_{11}$ \\
& $(820,929)$ & 1 & $(20,137)_{131}$ \\
\hline 953 & $(156,953)$ & 1 & $(2,7)_{5},(4,7)_{5},(2,29)_{14},(16,29)_{14},(18,43)_{25}$, \\
\hline 971 & $(166,971)$ & 42 & $(24,43)_{25},(36,43)_{25},(26,71)_{48},(56,71)_{48}$, \\
& & & $(6,211)_{127}$ \\
\hline 1061 & $(474,1061)$ & 1 & $(2,5)_{1}$ \\
\hline 1091 & $(888,1091)$ & 8 & $(2,5)_{1},(4,17)_{3},(8,17)_{3},(12,17)_{3},(8,41)_{25}$ \\
\hline 1117 & $(794,1117)$ & 1 & $(2,5)_{2}$ \\
\hline 1129 & $(348,1129)$ & 5 & $(2,11)_{7},(4,11)_{7},(6,11)_{7},(8,11)_{7},(20,41)_{22}$ \\
\hline 1151 & $(534,1151)$ & 1 & $(4,11)_{7}$ \\
& $(784,1151)$ & 1 & $(4,11)_{7}$ \\
& $(968,1151)$ & 1 & $(8,1)_{7}$ \\
\hline 1153 & $(802,1153)$ & 1 & $(2,5)_{3}$ \\
\hline 1193 & $(262,1193)$ & 1 & $(2,5)_{3}$ \\
\hline 1201 & $(676,1201)$ & 1 & $(4,17)_{11}$ \\
\hline 1217 & $(784,1217)$ & 3 & $(4,13)_{8},(8,13)_{8},(48,97)_{53}$ \\
& $(866,1217)$ & 1 & $(2,17)_{10}$ \\
& $(1118,1217)$ & 3 & $(2,13)_{8},(6,13)_{8},(10,13)_{8}$ \\
\hline 1229 & $(784,1229)$ & 28 & $(4,17)_{5},(8,17)_{5},(12,17)_{5},(16,29)_{11}$, \\
& & & $(32,113)_{99},(784,8597)_{1229},(2012,8597)_{1229}$, \\
& & & $(3240,8597)_{1229},(5696,8597)_{1229}$, \\
\hline 1237 & $(874,1237)$ & 1 & $(2,5)_{2}$ \\
\hline 1279 & $(518,1279)$ & 1 & $(2,7)_{5}$ \\
\hline
\end{tabular}


Table 3: Irregular pairs $(r, p)$ along with the corresponding helpful pairs $\left(t_{j}, q_{j}\right)_{a\left(\bmod q_{j}\right)}$ satisfying the conditions of Proposition 4

\begin{tabular}{|c|c|c|c|}
\hline$a$ & irregular pair $(r, p)$ & $\overline{\ell_{r}}$ & helpful pairs $\left(t_{j}, q_{j}\right)_{a\left(\bmod q_{j}\right)}$ \\
\hline 1283 & $(510,1283)$ & 10 & $\begin{array}{l}(2,5)_{3},(2,11)_{7},(4,11)_{7},(6,11)_{7},(8,11)_{7} \\
(6920,12821)_{1283}\end{array}$ \\
\hline 1291 & $\begin{array}{l}(206,1291) \\
(824,1291)\end{array}$ & $\begin{array}{l}2 \\
1\end{array}$ & $\begin{array}{l}(2,5)_{1},(56,61)_{10} \\
(14,31)_{20}\end{array}$ \\
\hline 1297 & $\begin{array}{l}(202,1297) \\
(220,1297) \\
\end{array}$ & $\begin{array}{l}1 \\
1\end{array}$ & $\begin{array}{l}(2,5)_{2} \\
(12,17)_{5}\end{array}$ \\
\hline 1301 & $(176,1301)$ & 1 & $(20,53)_{29}$ \\
\hline 1307 & $\begin{array}{l}(382,1307) \\
(852,1307) \\
\end{array}$ & $\begin{array}{l}3 \\
3 \\
\end{array}$ & $\begin{array}{l}(2,7)_{5},(4,7)_{5},(2994,3919)_{1307} \\
(2,7)_{5},(4,7)_{5},(852,3919)_{1307}\end{array}$ \\
\hline 1319 & $\overline{(304,1319)}$ & 360 & $\begin{array}{l}(2,7)_{3},(4,7)_{3},(8,17)_{10},(6,31)_{17},(12,31)_{17} \\
(18,31)_{17},(24,31)_{17},(6,37)_{24},(12,37)_{24}, \\
(18,37)_{24},(30,73)_{5},(36,73)_{5},(66,73)_{5} \\
(2940,11863)_{1319},(42480,52721)_{1319}\end{array}$ \\
\hline 1327 & $(466,1327)$ & 2 & $(2,5)_{2},(4,13)_{1}$ \\
\hline 1367 & $(234,1367)$ & 6 & $(2,5)_{2},(4,13)_{2},(8,13)_{2},(234,4099)_{1367}$ \\
\hline 1369 & $(32,37)$ & 10 & $(2,11)_{5},(6,11)_{5},(14,31)_{5},(8,41)_{16},(20,61)_{27}$ \\
\hline 1381 & $(266,1381)$ & 1 & $(2,5)_{1}$ \\
\hline 1409 & $(358,1409)$ & 1 & $(6,23)_{6}$ \\
\hline 1429 & $(996,1429)$ & 1 & $(44,239)_{234}$ \\
\hline 1439 & $(574,1439)$ & 630 & $\begin{array}{l}(4,11)_{9},(8,11)_{9},(2,19)_{14},(4,19)_{14},(8,19)_{14}, \\
(10,19)_{14},(12,19)_{14},(2,31)_{13},(6,31)_{13}, \\
(12,31)_{13},(22,31)_{13},(26,31)_{13},(24,37)_{33}, \\
(6,43)_{20},(24,43)_{20},(10,61)_{36},(30,61)_{36}, \\
(40,61)_{36},(30,71)_{19},(40,71)_{19},(36,127)_{42}, \\
(16,181)_{172},(50,181)_{172},(140,181)_{172}, \\
(60,211)_{173},(574,8629)_{1439},(50904,60397)_{1439}\end{array}$ \\
\hline 1483 & $(224,1483)$ & 2 & $(2,13)_{1},(8,13)_{1}$ \\
\hline 1499 & $(94,1499)$ & 24 & $\begin{array}{l}(2,7)_{1},(4,7)_{1},(6,13)_{4},(4,17)_{3},(8,17)_{3} \\
(10,17)_{3},(12,17)_{3},(14,17)_{3},(736,857)_{642}\end{array}$ \\
\hline
\end{tabular}




\section{REFERENCES}

[1] T. Arakawa, T. Ibukiyama and M. Kaneko, Bernoulli numbers and zeta functions, Springer Monographs in Mathematics, Springer Japan, 2014.

[2] E. Bach, D. Klyve and J. Sorenson, Computing prime harmonic sums, Math. Comp. 78 (2009), $2283-2305$.

[3] J. M. Borwein, A. van der Poorten, J. Shallit and W. Zudilin, Neverending Fractions, An Introduction to Continued Fractions, Australian Mathematical Society Lecture Series 23 (2014), Cambridge University Press.

[4] J. Büthe and P. Moree, Forbidden integer ratios of consecutive power sums. II, in preparation.

[5] W. Butske, L. M. Jaje and D. R. Mayernik, On the equation $\sum_{p \mid N}(1 / p)+(1 / N)=1$, pseudoperfect numbers, and perfectly weighted graphs, Math. Comp. 69 (2000), 407-420.

[6] L. Carlitz, Note on irregular primes, Proc. Amer. Math. Soc. 5 (1954), 329-331.

[7] Y. Gallot, P. Moree and W. Zudilin, The Erdős-Moser equation $1^{k}+2^{k}+\cdots+(m-1)^{k}=m^{k}$ revisited using continued fractions, Math. Comp. 80 (2011), 1221-1237.

[8] K. Ireland and M. Rosen, A classical introduction to modern number theory, Springer-Verlag, New York, 1990.

[9] B. C. Kellner, Über irreguläre Paare höherer Ordnungen, Diplomarbeit, Mathematisches Institut der Georg-August-Universität zu Göttingen, Germany, 2002. (Also available at http://www . bernoulli.org/ bk/irrpairord.pdf)

[10] B. C. Kellner, On irregular prime power divisors of the Bernoulli numbers, Math. Comp. 76 (2007), 405-441.

[11] B. C. Kellner, On stronger conjectures that imply the Erdős-Moser conjecture, J. Number Theory 131 (2011), 1054-1061.

[12] E. E. Kummer, Allgemeiner Beweis des Fermatschen Satzes, dass die Gleichung $x^{\lambda}+y^{\lambda}=z^{\lambda}$ durch ganze Zahlen unlösbar ist, für alle diejenigen Potenz-Exponenten $\lambda$, welche ungerade Primzahlen sind und in den Zählern der ersten $(\lambda-3) / 2$ Bernoullischen Zahlen als Factoren nicht vorkommen, J. Reine Angew. Math. 40 (1850), 131-138.

[13] M. Lang, Internship report: my studies on the Erdős-Moser equation $1^{k}+2^{k}+\cdots+(m-1)^{k}=$ $m^{k}, 2010$, unpublished.

[14] F. Luca, A. Pizarro-Madariaga and C. Pomerance, On the counting function of irregular primes, Indag. Math. 26 (2015), 147-161.

[15] T. Metsänkylä, Distribution of irregular prime numbers, J. Reine Angew. Math. 282 (1976), $126-130$.

[16] P. Moree, On a theorem of Carlitz-von Staudt, C. R. Math. Rep. Acad. Sci. Canada 16 (1994), $166-170$.

[17] P. Moree, Diophantine equations of Erdős-Moser type, Bull. Austral. Math. Soc. 53 (1996), 281-292.

[18] P. Moree, A top hat for Moser's four mathemagical rabbits, Amer. Math. Monthly 118 (2011), 364-370.

[19] P. Moree, Moser's mathemagical work on the equation $1^{k}+2^{k}+\cdots+(m-1)^{k}=m^{k}$, Rocky Mountain J. Math. 43 (2013), 1707-1737.

[20] P. Moree, Counting numbers in multiplicative sets: Landau versus Ramanujan, Šiauliai Math. Semin. 8 (16) (2013), 161-179.

[21] P. Moree, H. te Riele and J. Urbanowicz, Divisibility properties of integers $x, k$ satisfying $1^{k}+\cdots+(x-1)^{k}=x^{k}$, Math. Comp. 63 (1994), 799-815.

[22] L. Moser, On the diophantine equation $1^{n}+2^{n}+3^{n}+\cdots+(m-1)^{n}=m^{n}$. Scripta Math. 19 (1953). 84-88.

[23] P. Ribenboim, Catalan's conjecture. Are 8 and 9 the only consecutive powers? Academic Press, Inc., Boston, MA, 1994.

[24] W. Schwarz and J. Spilker, Arithmetical functions, London Mathematical Society Lecture Note Series 184, Cambridge University Press, Cambridge, 1994. 
[25] C. L. Siegel, Zu zwei Bemerkungen Kummers, Nachr. Akad. Wiss. Göttingen Math.-Phys. Kl. II 1964 (1964), 51-57.

[26] P. Tegelaar, Handwritten comments on an earlier version of this paper, March 6, 2015.

Department of Mathematics, Moscow State Pedagogical University, KrasnoPRUDNAYA STR. 14, Moscow 107140, Russia

E-mail address: jbaulina@mail.ru

Max-Planck-Institut für Mathematik, Vivatsgasse 7, D-53111 Bonn, Germany

E-mail address: moree@mpim-bonn.mpg.de 